\title{
Optimising $\mathrm{T}$ cell (re)boosting strategies for adenoviral and modified vaccinia Ankara vaccine regimens in humans
}

\author{
Stefania Capone (iD ${ }^{1}$, Anthony Brown ${ }^{2}$, Felicity Hartnell ${ }^{2}$, Mariarosaria Del Sorbo ${ }^{1}$, Cinzia Traboni ${ }^{1,9}$, Ventzislav Vassilev ${ }^{3}$, \\ Stefano Colloca ${ }^{1}$, Alfredo Nicosia ${ }^{4,5,6}$, Riccardo Cortese ${ }^{1}$, Antonella Folgori ${ }^{1}$, Paul Klenerman ${ }^{2,7,8}$, Eleanor Barnes ${ }^{2,7,8,11}$ and \\ Leo Swadling (iD $^{2,10,11 凶}$
}

Simian adenoviral and modified vaccinia Ankara (MVA) viral vectors used in heterologous prime-boost strategies are potent inducers of $T$ cells against encoded antigens and are in advanced testing as vaccine carriers for a wide range of infectious agents and cancers. It is unclear if these responses can be further enhanced or sustained with reboosting strategies. Furthermore, despite the challenges involved in MVA manufacture dose de-escalation has not been performed in humans. In this study, healthy volunteers received chimpanzee-derived adenovirus-3 and MVA vaccines encoding the non-structural region of hepatitis $C$ virus (ChAd3-NSmut/MVA-NSmut) 8 weeks apart. Volunteers were then reboosted with a second round of ChAd3-NSmut/MVA-NSmut or MVA-NSmut vaccines 8 weeks or 1-year later. We also determined the capacity of reduced doses of MVA-NSmut to boost ChAd3NSmut primed T cells. Reboosting was safe, with no enhanced reactogenicity. Reboosting after an 8-week interval led to minimal reexpansion of transgene-specific T cells. However, after a longer interval, $\mathrm{T}$ cell responses expanded efficiently and memory responses were enhanced. The 8-week interval regimen induced a higher percentage of terminally differentiated and effector memory T cells. Reboosting with MVA-NSmut alone was as effective as with ChAd3-NSmut/MVA-NSmut. A ten-fold lower dose of MVA $\left(2 \times 10^{7} \mathrm{pfu}\right)$ induced high-magnitude, sustained, broad, and functional Hepatitis $C$ virus (HCV)-specific T cell responses, equivalent to standard doses $\left(2 \times 10^{8} \mathrm{pfu}\right)$. Overall, we show that following Ad/MVA prime-boost vaccination reboosting is most effective after a prolonged interval and is productive with MVA alone. Importantly, we also show that a ten-fold lower dose of MVA is as potent in humans as the standard dose.

npj Vaccines (2020)5:94; https://doi.org/10.1038/s41541-020-00240-0

\section{INTRODUCTION}

Diverse vaccine modalities have been tested in the past in an attempt to recapitulate the T cell mediated life-long immunity that can be induced upon acute-resolving viral infection. Great advances in the field of molecular virology have enabled the manipulation of viruses as vectors to deliver foreign antigens and these have emerged as the most potent method of inducing an antigen-specific T cell response (reviewed in refs. ${ }^{1,2}$ ). These viruses have highly evolved mechanisms of cell entry and protein expression that can be exploited for the delivery of selected immunogenic antigens, whilst serial passage (in the case of modified vaccinia Ankara ${ }^{3}$ ) or gene deletions (for adenoviruses) can render these viral vectors non-pathogenic and non-replicative.

The best characterized viral vectors and the most widely used in current phase I and II clinical trials are Adenoviral (Ad) vectors and MVA (World Health Organisation International Clinical Trials Registry, http://apps.who.int/trialsearch ${ }^{1}$ ). $\mathrm{Ad}^{4,5}$ and $M V A^{6-8}$ vectors are being developed as candidate vaccines for a diverse range of pathogens, including several prime-boost regimens making use of Ad vector's propensity to prime a strong T cell response (MERS ${ }^{9}$; SARS-Cov- $\left.2^{10}\right)$, and MVA's ability to broaden and enhance both peak and memory transgene-specific $T$ cell responses $\left(\mathrm{HCV}^{11}\right.$; Influenza ${ }^{12}$; Ebola ${ }^{13} ;$ Malaria $^{8} ; \mathrm{RSV}^{14} ;$ HIV $^{15,16}$ ). Ad/MVA primeboost regimens are not only highly immunogenic, but have shown encouraging efficacy in animal and human challenge models
(Ebola ${ }^{17}$, Malaria $\left.{ }^{18}, \mathrm{SIV}^{19}, \mathrm{RSV}^{20}\right)$. Although Ad and MVA vectors used in prime-boost strategies are both potent and safe, few studies have investigated whether reboosting strategies may be employed to further enhance immunogenicity, whilst also retaining their safety and minimal reactogenicity ${ }^{6,21,22}$.

We have developed a T cell-based vaccine regimen for Hepatitis $C$ virus (HCV), employing a chimpanzee-derived adenovirus 3 (ChAd3) prime and an MVA boost encoding the non-structural region of HCV (NSmut; genotype $1 \mathrm{~b}$ ), which was the first vaccine for HCV to progress to Phase II efficacy testing (NCT01436357). Although, we have shown these vaccines to be highly immunogenic, inducing high-magnitude polyfunctional $\mathrm{CD}^{+}$and $\mathrm{CD} 8^{+}$ T cells ${ }^{11}$, recent analysis (NCT01436357) showed that this regimen was not effective in preventing chronic infection in intravenous drug using populations. Therefore, new strategies are required to enhance and/or sustain antiviral immunity.

In this study, we describe the results of a phase I clinical trial in which we investigated the impact of reboosting and dose deescalation on the magnitude and quality of the $T$ cell response induced in humans, using our candidate HCV vaccine as a model vaccine. Specifically, we assess the impact of a second round of ChAd3 and MVA vaccinations, or MVA vaccination alone, given after a short or long interval following initial ChAd3/MVA primeboost.

\footnotetext{
${ }^{1}$ ReiThera Srl, Via di Castel Romano, 100, 00128 Rome, Italy. ${ }^{2}$ Nuffield Department of Medicine, University of Oxford, Oxford, UK. ${ }^{3}$ GSK Vaccines, Brussels, Belgium. ${ }^{4}$ Keires AG,

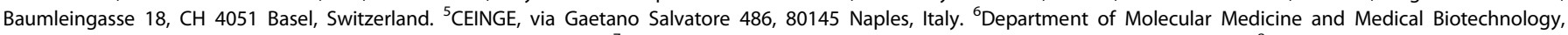

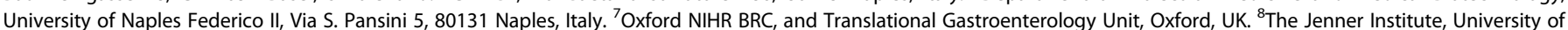
Oxford, Oxford, UK. ${ }^{9}$ Present address: Nouscom Srl, Via di Castel Romano, 100, 00128 Rome, Italy. ${ }^{10}$ Present address: Rayne Institute, University College London, London, UK. ${ }^{11}$ These authors jointly supervised this work: Eleanor Barnes, Leo Swadling. ${ }^{凶}$ email: I.swadling@ucl.ac.uk
} 
Additionally, since MVA vaccine manufacture is challenging and using effective lower doses could increase the number of vials that are produced in this process, so benefiting large-scale vaccine programs $^{23}$, we perform a comprehensive analysis of the HCVspecific T cells response induced by lower doses of MVA boost vaccination.

\section{RESULTS}

Reboosting vaccinations with ChAd3-NSmut and MVA-NSmut are safe and well tolerated

A primary endpoint for this phase-I clinical trial was to assess the safety and tolerability of secondary administration of Ad and MVA vectors in healthy volunteers. The volunteer arm protocols are described in Fig. 1 and Supplementary Table 1 and volunteer demographics in Supplementary Table 2. Following ChAd3-NSmut vaccination (whether first or second administrations) most adverse events (AEs; 97.5\%) were mild or moderate and there were no serious adverse reactions (Supplementary Fig. 1 and Supplementary Table 3). As previously described ${ }^{11,14}$, AEs of all severities were more common after MVA-NSmut (chi-square $p=0.0468$; Supplementary Table 3). Importantly, in volunteers who were revaccinated (arms A3-A5) with a second round of ChAd3 and/or MVA, reboosting vaccinations resulted in a similar AE profile to the first round of vaccinations regardless of time delay between vaccinations (8-92 weeks; Supplementary Fig. 1; Supplementary Table 3). Overall, both ChAd3-NSmut and MVA-NSmut were well tolerated regardless of the number of administrations, the order in which they were administered, or the time between vaccinations.

The magnitude of the peak and memory vaccine-induced HCVspecific $T$ cell response is improved when the interval before reboosting is prolonged

We assessed whether the response to vaccination could be enhanced in magnitude or functionality by administering a second cycle of ChAd3-NSmut/MVA-NSmut vaccination after a short (8 weeks- Arm A3) or long time interval (39-84 weeks- Arm A4; volunteers re-enrolled from initial clinical trial of ChAd3NSmut/MVA-NSmut, see methods; Fig. 1). When all four vaccines were given 8 weeks apart (Fig. 2a; Arm A3) no expansion of HCV NSmut-specific T cells was seen after the second ChAd3-NSmut vaccination in all five volunteers and only a weak expansion was seen after the second MVA-NSmut; however, when there was a long interval of 39-84 weeks between the first and second rounds of ChAd3-NSmut/MVA-NSmut (Arm A4; Fig. 2b) HCV-specific T cell responses expanded in three of the four volunteers to levels comparable to those seen in the initial round of vaccinations. The relative fold change in $\mathrm{HCV}$-specific $\mathrm{T}$ cell response after vaccination ( 2 weeks post-ChAd3-NSmut and 1 week post-MVANSmut) was on average higher when a longer interval was used (Fig. 2c), but only reached significance after reboosting with ChAd3-NSmut (Fig. 2d).

To investigate whether reboosting with MVA-NSmut alone would be as effective as reboosting with ChAd3/MVA, 5 volunteers in Arm A5 received a second MVA-NSmut 32 weeks after their first MVA-NSmut boost (Fig. 2e). The peak magnitude of the HCVspecific $T$ cells response after the second MVA vaccination did not differ when it was preceded by a second ChAd3-NSmut vaccination or was given alone (Fig. 2d, e; peak post second MVA-NSmut vaccination arm A4 median 3384 range 385-6260, arm A5 median 2532 range 832-3599, $p=0.6857$ ).

We next assessed whether reboosting impacted on the breadth of the T cell response and the immunodominance of different HCV antigens encoded within the vaccine. Vaccination-induced T cells targeting all HCV non-structural proteins encoded within the vaccines and the relative contribution of each HCV antigen was relatively unchanged from post-prime vaccination to boosting with MVA and subsequent reboosting with ChAd3-NSmut and MVA-NSmut (Fig. 2f, Supplementary Fig. 3).

The functionality of HCV-specific T cells induced by different vaccine regimens was tested at the time of peak magnitude after vaccination by ex vivo intracellular cytokines staining. For all cytokines tested (IFN $\gamma$, TNFa, and IL-2) the magnitude of the CD4 ${ }^{+}$ and $\mathrm{CD}^{+}$cytokine-producing $\mathrm{T}$ cell response was larger when a longer interval was left between rounds of vaccination (arm A4: red > arm A3: green), in agreement with the IFNY ELISpot data (Fig. 3). When reboosting after a long interval with a second MVANSmut vaccination $T$ cell responses were comparable whether a second ChAd-NSmut was given before or not (arm A4 red vs. arm A5 blue). Overall $\mathrm{CD}^{+} \mathrm{T}$ cell responses were lower in magnitude than $\mathrm{CD}^{+} \mathrm{T}$ cell responses.

We next used boolean gating to assess whether the proportion of polyfunctional transgene-specific T cells, capable of producing IFNY, TNFa, and IL-2 in combination, differ at the peak of the T cell response between different reboosting regimens. We have previously shown that polyfunctional T cells induced by ChAd3NSmut/MVA-NSmut vaccination, that are capable of producing IFN $\gamma$, TNFa, and IL-2 in combination, produce a larger amount of each individual cytokine on a per cell basis, which may indicate greater antiviral activity 9 . As has been previously described, HCV NS-specific $\mathrm{CD}^{+}{ }^{+} \mathrm{T}$ cells were more polyfunctional than $\mathrm{CD}^{+}$ owing to their greater propensity to produce IL2 (Fig. 3d). After ChAd3-NSmut prime and MVA-NSmut boost the majority of transgene-specific $\mathrm{CD}^{+} \mathrm{T}$ cells were IFN $\gamma$ single producers; however, a larger proportion were polyfunctional after reboosting

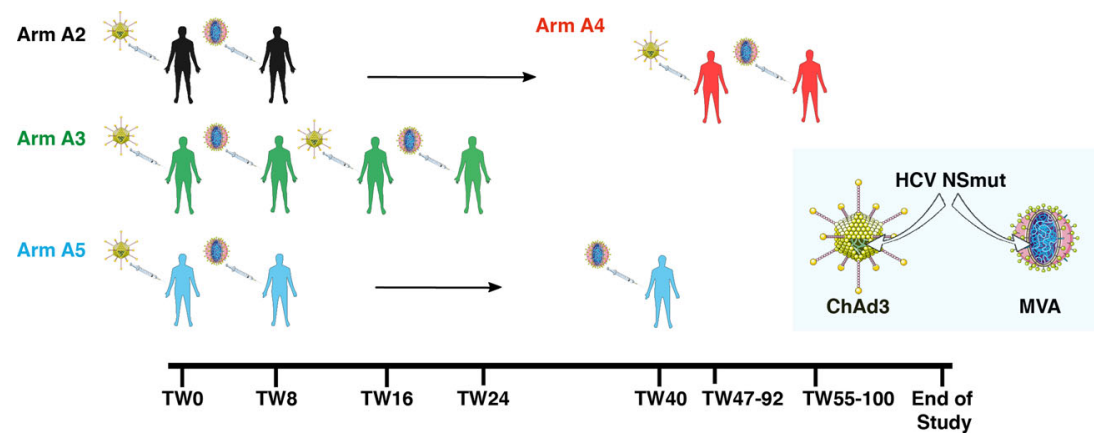

Fig. 1 Reboosting vaccination schedules. A chimpanzee-derived adenovirus 3 (ChAd3) and a modified vaccinia Ankara (MVA) were engineered to express the non-structural region (NS3-5b, BK strain genotype $1 \mathrm{~b}$, NSmut) of hepatitis $C$ virus. Healthy volunteers were given a prime-boost vaccine regimen (ChAd3-NSmut at trial week 0 [TW0], MVA-NSmut boost at TW8) followed by reboosting vaccination with a second round of ChAd3-NSmut/MVA-NSmut after a short interval (all vaccines 8 weeks apart, arm A3), after a long interval (TW47-92, arm A4; volunteers re-recruited from arm A2), or were reboosted with MVA-NSmut alone after a long interval (TW40, arm A5). Vaccine dose $2.5 \times 10^{10}$ viral particles (vp) for ChAd3-NSmut and $2 \times 10^{8}$ plaque forming units (pfu) for MVA-NSmut. 
A

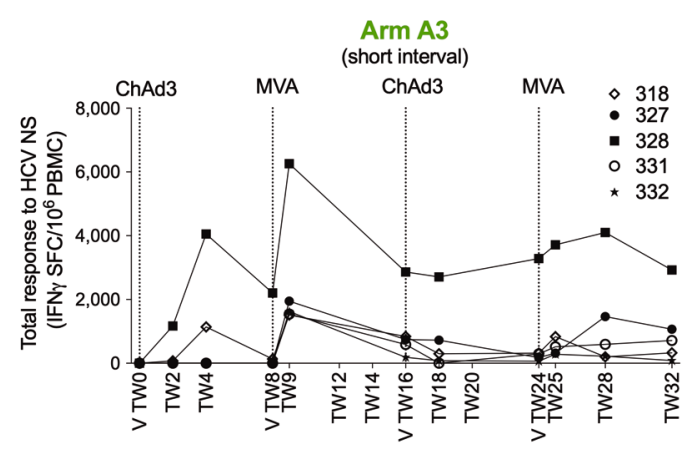

C

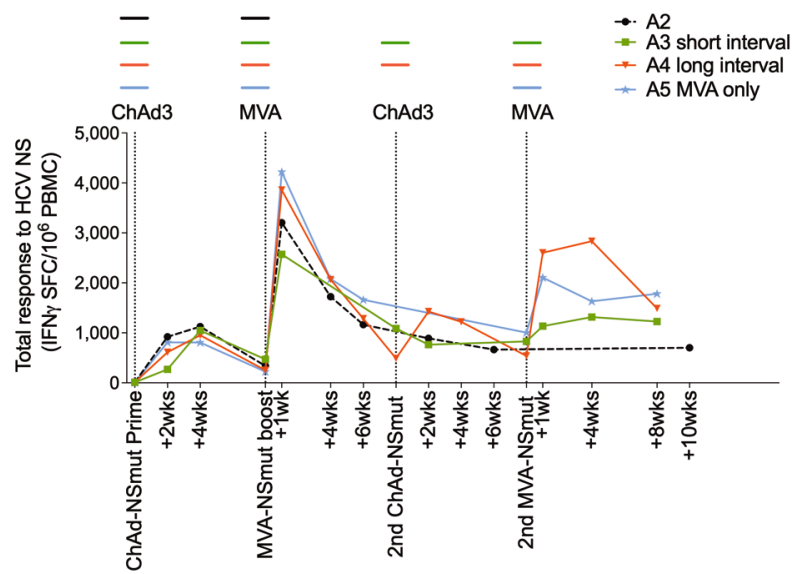

E

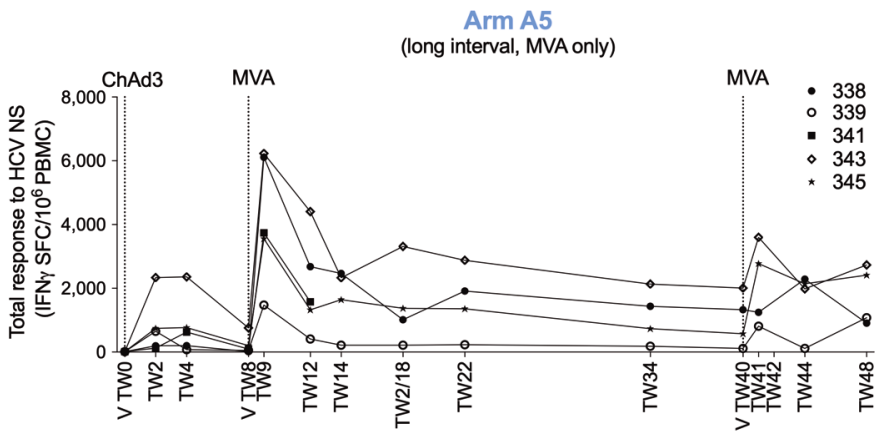

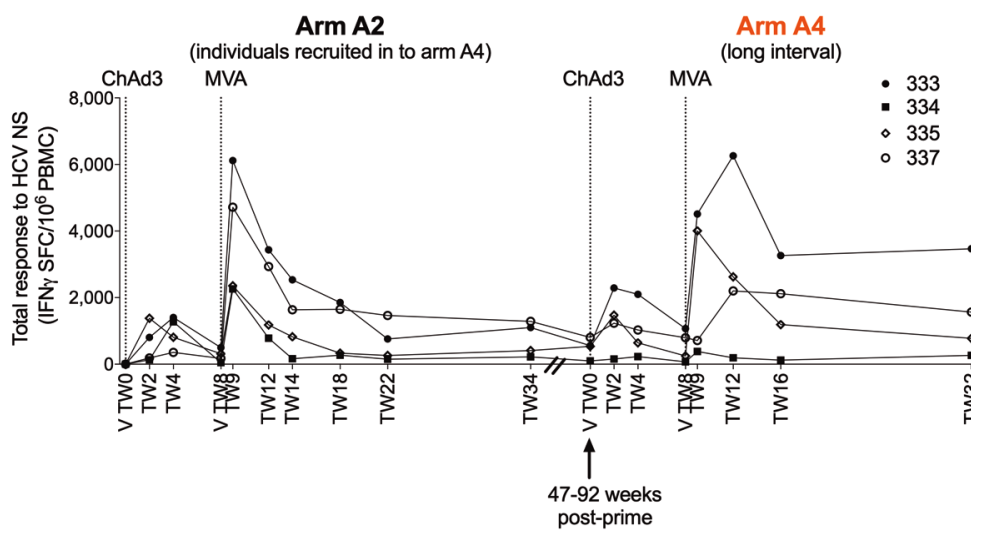

D

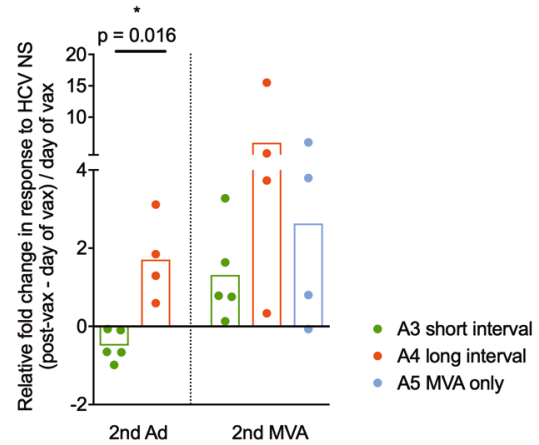

$\mathbf{F}$

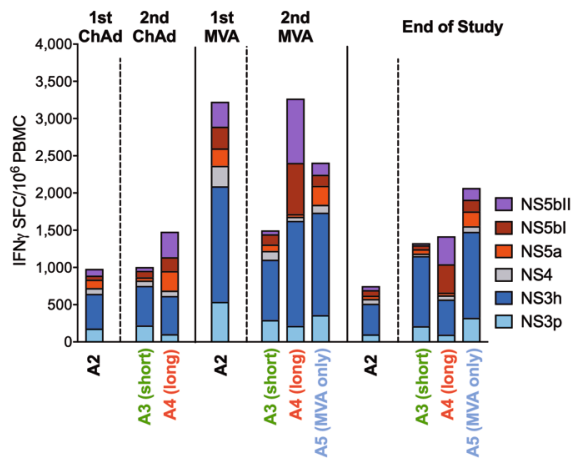

Fig. 2 HCV-specific T cell responses are expanded by reboosting after a long interval with a second round of ChAd3-NSmut and MVANSmut or with MVA-NSmut alone. a-c The total ex vivo T cell response to the non-structural (NS) region of HCV encoded within the vaccine is shown over time (IFN $\gamma$ ELISpot; spot forming cells per $10^{6} \mathrm{PBMC}$; calculated by summing the responses to positive peptide pools corrected for background; materials and methods). Individual healthy volunteers are shown for arms A3 (a), arm A2/A4 (b) and overlaid group means (c). Colored horizontal lines above the graph indicate when a vaccine was given. $\mathbf{d}$ Fold change in T cell response to HCV NS (post-vaccination response-pre-vaccination response/pre-vaccination response). Post-vaccination response was taken as +2 weeks for ChAd3, +1 week for MVA. Bars at group mean. e The total ex vivo T cell response to the NS region of HCV over time for individual volunteers for arm A5. a-c, e Vertical dashed lines indicate time of vaccination. chAd3, chimpanzee-derived adenovirus 3; MVA, modified vaccina Ankara. $\mathbf{f}$ The ex vivo T cell response to HCV by antigen at the peak of the T cell response post-vaccination and at the end of the study (2-4 weeks post-ChAd3-NSmut and 1-4 weeks post-MVA-NSmut for peak response and 14-40 weeks post vaccination for end of study; group mean per peptide pool by IFN $\gamma$ ELISpot, $n=4-9$; all pools background subtracted).

regardless of the regimen used, in particular producing both IFNy and TNFa. A larger population of $\mathrm{CD}^{+} \mathrm{T}$ cells producing all three cytokines was seen when reboosting with a further round of ChAd3-NSmut/MVA-NSmut (arm A4) or MVA-NSmut alone (arm A5) after a longer interval.
In summary, HCV-specific T cell responses were most effectively expanded and showed greatest polyfunctionality when individuals were re-vaccinated after an extended period of time, with a single second MVA vaccination as effective as re-vaccination with both Ad and MVA 
A

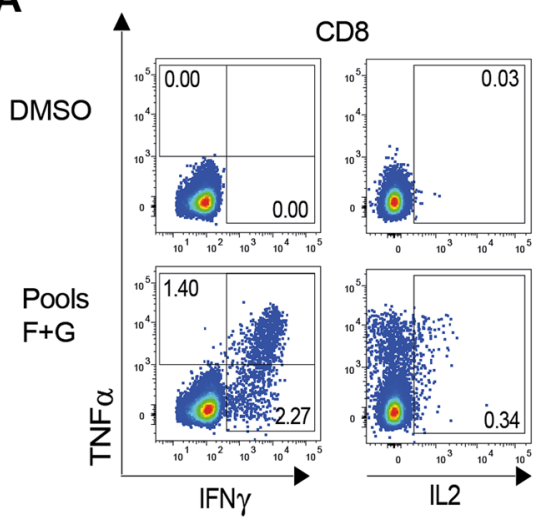

B

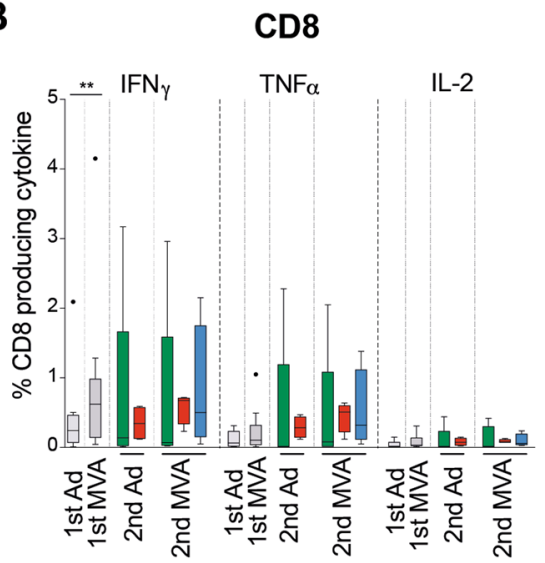

CD4
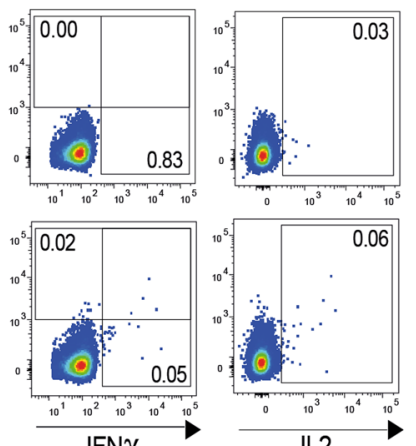

IL2
C

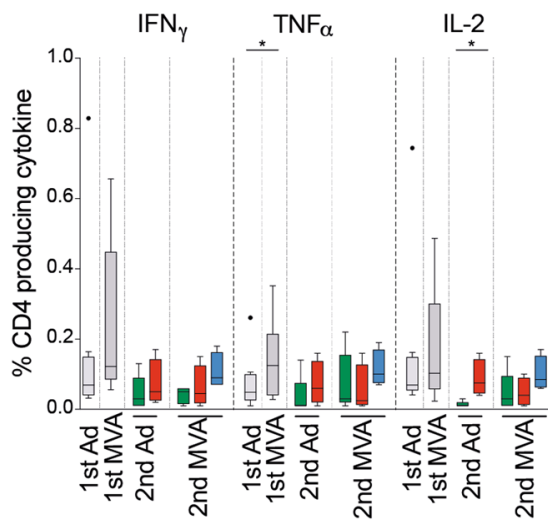

$\square$ A2 Ad/MVA

A3 Ad/MVA/Ad/MVA (short)

- A4 Ad/MVA_Ad/MVA (long)

A5 Ad/MVA MVA

D

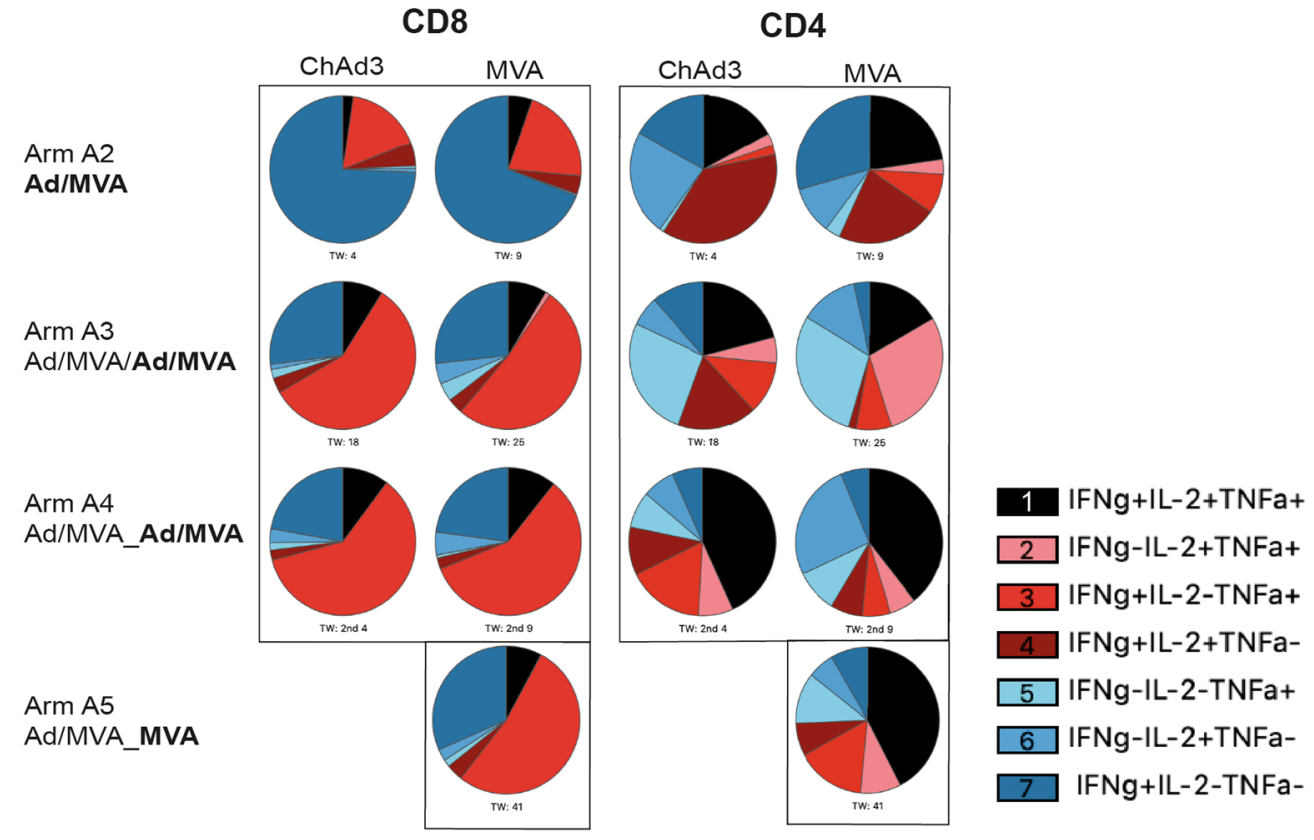

Monitoring anti-vector humoral and $\mathrm{T}$ cell responses

To evaluate the impact of pre-existing and vaccination-induced antiChAd3 vector immunity on the ability of volunteers to respond to repeated vaccine administration volunteers were monitored for ChAd3 neutralizing antibody $(\mathrm{nAb})$ titers and for IFN $\gamma$ secretion upon
PBMC stimulation with a pool of overlapping peptides of the Ad5 Hexon protein sequence. Hexon is the most abundant $\mathrm{Ad}$ capsid protein and main target of $T$ cell responses, with many described $\mathrm{CD}^{+}$and $\mathrm{CD}^{+}$epitopes residing in protein regions highly conserved across human and primate adenoviral strains ${ }^{24-27}$. 
Fig. 3 Cytokine-producing HCV-specific T cells are induced by reboosting after a long interval. a-c Comparison of ex vivo cytokine production by $T$ cells at the time of peak magnitude of response (peak NS response by IFN $\gamma$ ELISpot) to each vaccination (1-4 weeks post-

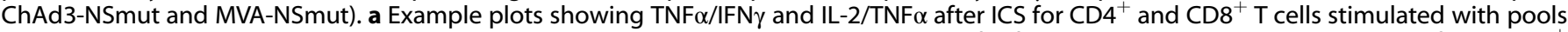
$\mathrm{F}+\mathrm{G}$ or DMSO control (volunteer 343 arm A5 TW41, 2nd MVA; see Supplementary Fig. 4 for full gating strategy). The percentage of total CD8 ${ }^{+}$ (b) or $\mathrm{CD}^{+}{ }^{+}$(c) T cells producing IFN $\gamma$, TNF $\alpha$, or IL-2 after overnight stimulation with peptides covering the non-structural region of HCV within the vaccines (Pools $\mathrm{F}+\mathrm{G}, \mathrm{H}+\mathrm{l}, \mathrm{L}+\mathrm{M}$ summed after subtracting values for paired DMSO wells from each stimulation) are shown. d The proportion of the transgene-specific $\mathrm{CD}^{+}$or $\mathrm{CD}^{+} \mathrm{T}$ cells producing different combinations of IFN $\gamma$, TNF $\alpha$, and IL-2 are presented as pie charts (base mean) at the peak of the T cell response post vaccination (samples with a transgene-specific response $<0.025 \%$ of $C D 4^{+}$or $C D 8^{+}$ T cells were excluded from polyfunctionality analysis; $n=4-9$ per arm). b, c Box-whisker Tukey, outliers shown as dots ( $n=9$ A2, $n=5$ arm A3, $n=4$ arms A4, A5). Wilcoxon paired $t$ test 1 st Ad vs. 1st MVA, Mann-Whitney unpaired $t$ test for 2 nd Ad A3 vs. A4, Kruskal-Wallis one-way ANOVA with Dunn's correction for 2 nd MVA A3, A4, A5 per cytokine. Ad, adenovirus; MVA, modified vaccina Ankara. ${ }^{*} P<0.05 ;{ }^{* *} P<0.005$.

A

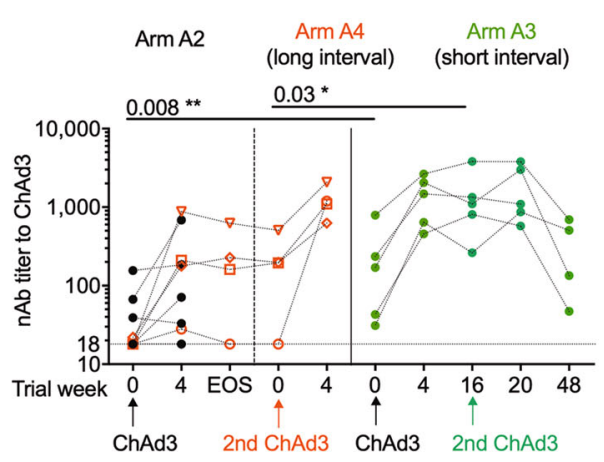

D

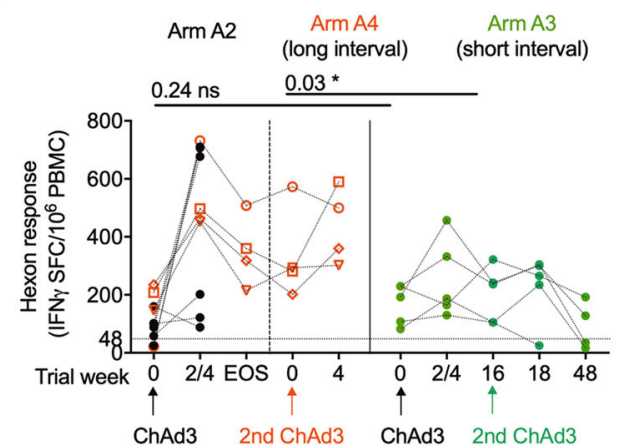

B
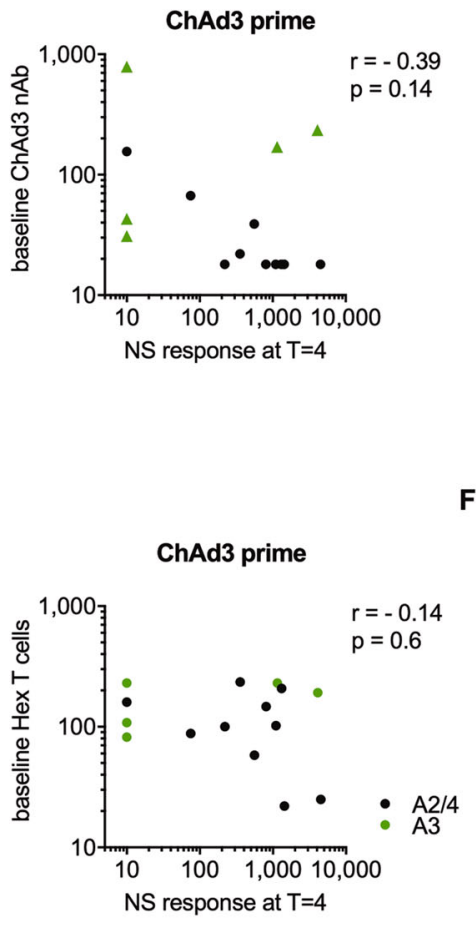
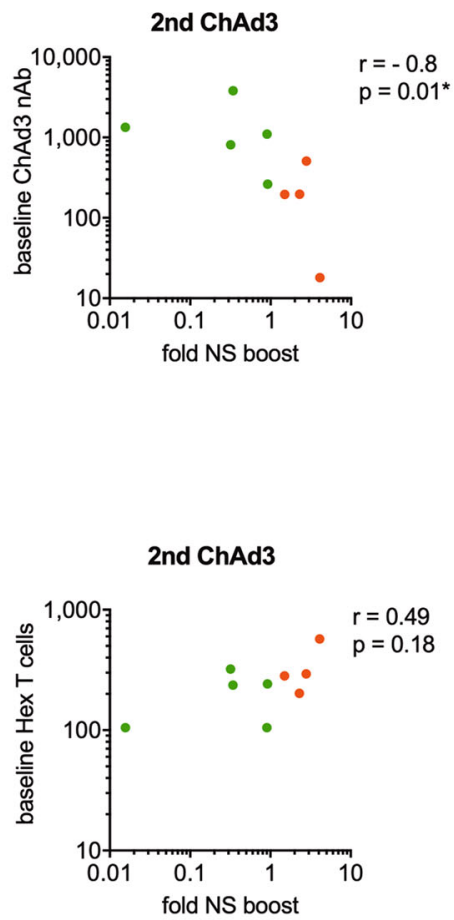

Fig. 4 Anti-vector immunity. a Titer of neutralising antibodies (nAbs) against ChAd3 vector (dotted line at nAb titer of 18, limit of detection for the assay). Volunteers who received ChAd3-NSmut prime and MVA-NSmut boost as part of arm A2 which were then reboosted in arm A4 are indicated by red symbols. Trial week indicates weeks since first vaccination for arm A2 and A3 and time of second ChAd3 vaccination for Arm A4. b Anti-ChAd3 nAb titer at baseline vs. peak magnitude of T cell responses to NS region of HCV post-vaccination (IFN $\gamma$ ELISpot; spot forming cells per $10^{6} \mathrm{PBMC}$ ). c Anti-ChAd3 nAb titer at time of second ChAd3-NSmut vaccination vs. fold change in magnitude of T cell responses to HCV NS post-vaccination. Fold change (FC) was calculated as (total NS response 2 weeks post-vaccination-NS response on day of second ChAd3-NSmut vaccination)/NS response pre-vaccination. d Ex vivo T cell response to Ad5 hexon (IFN $\gamma$ ELISpot; horizontal dotted line at 48, positivity cut-off for the assay). Trial week indicates weeks since first vaccination for arm A2 and A3 and time of second ChAd3 vaccination for Arm A4. e T cell response to Ad5 hexon at baseline vs. peak magnitude of T cell responses to NS region of HCV postvaccination. $\mathbf{f} \mathrm{T}$ cell response to Ad5 hexon at time of second ChAd3-NSmut vaccination vs. fold change in magnitude of T cell responses to HCV NS post vaccination (FC calculated as above). a, $\mathbf{d}$ Paired Wilcoxon $t$ test. b, $\mathbf{c}$, e, $\mathbf{f}$ Spearman rank correlation. ChAd3, chimpanzee-derived adenovirus 3; EOS, end of study. NS, non-structural; MVA, modified vaccina Ankara.

ChAd3 was selected as the candidate adenoviral vector due to the low global prevalence of pre-existing $n A b^{28}$; indeed this was found to be the case, with 12 of 14 individuals in groups $A 2$ and A3 having a baseline ChAd3 cross-reacting nAb titer below 200 (Fig. 4a) - a titer previously associated with reduced immunogenicity of Ad5 vectored vaccines ${ }^{29}$. The magnitude of the HCVspecific $\mathrm{T}$ cell response 4 weeks (peak) after primary ChAd vaccination did not correlate with baseline ChAd3 nAb titers (Fig. 4b). Baseline $n A b$ titers were overall higher in group $A 3$ compared to group A2 (GeoMean [GM] 133 vs. 28, $p=0.008^{* *}$ ), however, the vaccine-induced HCV-specific $\mathrm{T}$ cell response was not significantly different between arm A3 and A2 after ChAd priming ( $p=0.38$ ) or after the first MVA boost ( $p=0.42$; Fig. $2 a$ ). Overall, pre-existing $\mathrm{nAb}$ titers were of low magnitude and differences in pre-existing anti-vector immunity did not impact on vaccine immunogenicity.

Priming with the first $\mathrm{ChAd} 3$ vaccination resulted in an expansion of $\mathrm{nAb}$ in all but two individuals (Fig. 4a). However, $\mathrm{nAb}$ titers (that were higher at baseline in arm A3) were boosted to significantly higher levels in arm A3 than arms A2/A4, and, 
importantly, these titers remained significantly higher at the time of reboosting with a second ChAd3-NSmut vaccination (short interval gp A3 GM 1,037 vs. long interval gp A4 GM 137; $p=0.03$. Fig. 4a). Overall, the fold increase in HCV-specific T cell responses before and after 2nd ChAd3 administration negatively correlated with ChAd3 nAb titers at the time of vaccination (Fig. 4c), which suggests that high $\mathrm{nAb}$ titers at the time of reboosting with ChAd3-NSmut could have resulted in the observed poor/nonresponse to the encoded antigen after a 2 nd $\mathrm{ChAd} 3$ vaccination in group A3 (Fig. 2a).

In assessing the hexon-specific T cell response, no correlation with vaccine immunogenicity was seen. Baseline hexon $T$ cell responses were not significantly different between Arms $A 3$ and A4 (GM 155 vs. 89, $p=0.24$; Fig. 4d) and there was no correlation between the magnitude of the hexon-specific $T$ cell response at the time of ChAd3 administration and the induction (Fig. 4e) or expansion (Fig. 4f) of HCV-specific T cell response by vaccination in any groups. Taken together these data suggest that the humoral, rather than the $\mathrm{T}$ cell component, of the antiadenovirus response may interfere with re-administration of Ad vectors when anti-Ad antibodies reach high levels after Ad priming vaccination.

Reboosting changes the phenotype of the vaccine-induced memory $\mathrm{T}$ cell populations

We have previously shown that vaccine-induced $\mathrm{CD} 8^{+} \mathrm{T}$ cells were highly activated (as measured by CD38 and HLA-DR expression on MHC class I pentamer ${ }^{+}$T cells) immediately after ChAd3-NSmut prime and MVA-NSmut boost vaccinations. The strongest expression of activation markers on HCV-specific T cells coincides with the peak in magnitude of the response 1 week after MVA-NSmut vaccination ${ }^{11}$. We therefore investigated whether $T$ cell activation status correlates with the expansion of HCV-specific $\mathrm{T}$ cells response on reboosting. Only a small subset of HCV-specific $\mathrm{T}$ cells expressed activation markers after a second round of ChAd3-NSmut, irrespective of the time interval since the initial prime-boost vaccinations (Fig. 5). However, reboosting with a second MVA-NSmut vaccination, given alone or after a second ChAd3-NSmut, resulted in expression of activation markers on the majority of HCV-specific T cells (arm A4 or A5 vs. arm A3). Activation markers expression was rapidly lost with contraction of the HCV-specific T cell response, with little expression of these markers being seen in the resting memory populations at the end of the study. A higher expression of activation markers by HCVspecific $T$ cells was seen in all cases where an expansion of the HCV-specific T cell response occurred.

The co-expression pattern of the lymph node homing marker CCR7 and CD45 protein tyrosine phosphatase isoform CD45RA can be used to identify different memory subsets of $\mathrm{T}_{\text {cells }}{ }^{30}$. We costained HCV-specific T cells with these markers to investigate whether re-vaccination alters the proportions of $\mathrm{T}$ cell memory subsets across the trial. At the peak of the T cell response to initial ChAd3-NSmut prime and MVA-NSmut boost vaccinations HCVspecific $T$ cells were a mixed population of effector memory (Tem; $\mathrm{CD} 5 \mathrm{RA}^{-} \mathrm{CCR7}^{-}$) and central memory (Tcm; CD45RA ${ }^{-} \mathrm{CCR7}^{+}$) subsets (Fig. 6). When re-vaccinating with ChAd3-NSmut after a short (A3) or long (A4) interval most HCV-specific T cells were Tem and Temra (terminally differentiated effector memory, $\mathrm{CD}^{2} 5 \mathrm{RA}^{+} \mathrm{CCR7}^{-}$), with few Tcm. The intensive vaccination regimen of ChAd3-NSmut/MVA-NSmut/ChAd3-NSmut/MVA-NSmut all 8 weeks apart lead to a larger proportion of Temra and Tem T cells with a concomitant reduction in Tcm and naive-like memory $\mathrm{T}$ cells when compared to other regimens (Fig. 6a, b). Revaccination with a second MVA-NSmut also tended to produce more Tem, and fewer Tcm and Naïve-like T cells, similar to the proportions seen in memory responses to CMV (Fig. 6b) ${ }^{31}$. Overall, where a stronger T cell expansion was seen, as in Arm A4 and A5, a larger Tem population was seen immediately after vaccination, and reboosting lead to a more terminally differentiated population of $\mathrm{T}$ cells.

Co-expression patterns of the T-box transcription factors (TF), Tbet and Eomesodermin (Eomes) coordinate both $\mathrm{T}$ cell memory formation and effector function, in particular IFNY expression and cytolytic potential ${ }^{32-35}$. We assessed their expression on vaccineinduced HCV-specific T cells to see if re-vaccination drives a TF profile indicative of terminal differentiation or exhaustion. After all vaccinations, HCV-specific T cells were dominated by $\mathrm{Tbet}^{+}$(T-box transcription factor TBX21) Eomes $^{+}$(Eomesodermin) $\mathrm{T}$ cells, indicative of Tem and Temra ${ }^{31}$ subsets, with few Eomes ${ }^{-}$Tbet $^{-}$ (Tcm and Naïve-like), in line with surface marker expression (Fig. 6c, d). Vaccine-induced $T$ cells showed similar TF co-expression to functional memory $\mathrm{T}$ cells seen after CMV infection (Fig. 6c, d). There were few differences in Tbet and Eomes expression between
A

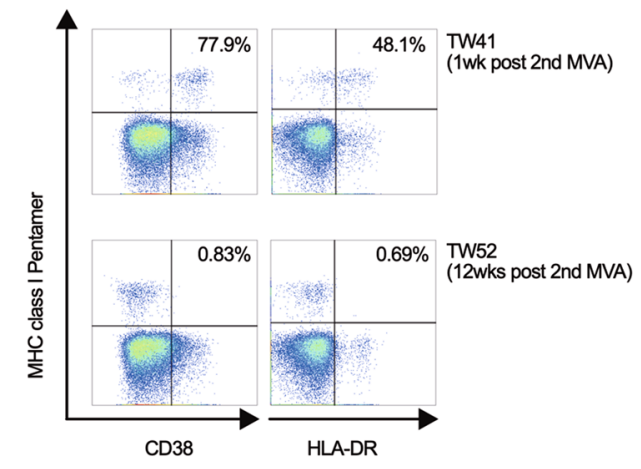

B

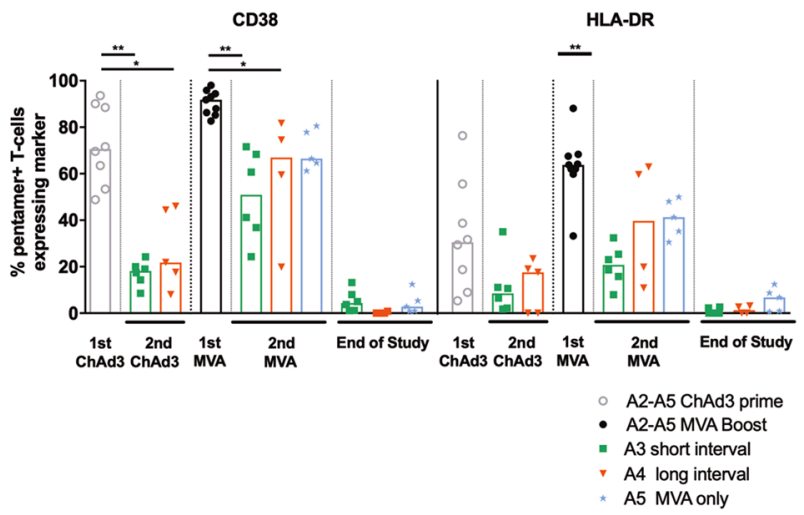

Fig. $5 \mathrm{~T}$ cell activation is strongest after MVA vaccination but it is not affected by the length of the interval since last NS encoding vaccination. a Example plots (CD8 ${ }^{+} \mathrm{T}$ cells) showing pentamer vs. activation marker (arm A5) 1 week and 12 weeks post second MVA-NSmut vaccination (see Supplementary Fig. 4 for full gating strategy). Percentage of pentamer ${ }^{+}$cells expressing the activation marker is shown. $\mathbf{b}$ Ex vivo $\mathrm{MHC}$ class I pentamer staining was performed on all HLA-A*01 ${ }^{+}$or $\mathrm{A}^{*} 02^{+}$individuals (NS3 ${ }_{1435-1443}, \mathrm{NS3}_{1406-1415}$, NS3 $3_{-1073-1081}$ see methods) from arms A2-A5. PBMC were co-stained with individual pentamers and antibodies against the CD8 ${ }^{+} \mathrm{T}$ cell surface activation markers CD38 and HLA-DR. The percentage of pentamer ${ }^{+}$cells expressing these markers is shown, bars at median. b Kruskal-Wallis one-way ANOVA with Dunn's correction comparing all group means for 1st and 2nd ChAd3-NSmut vaccination, and for 1st and second MVA vaccination for CD38 and for HLA-DR, significant differences shown. ${ }^{*} P<0.05$; ${ }^{* *} P<0.005$. 
A

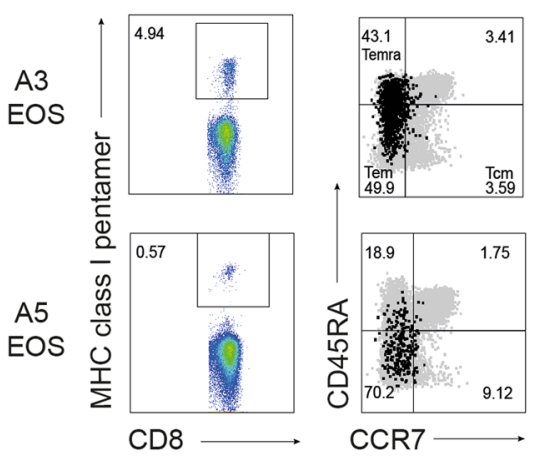

C

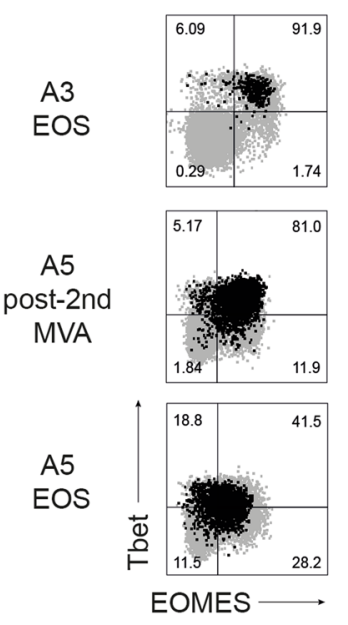

B

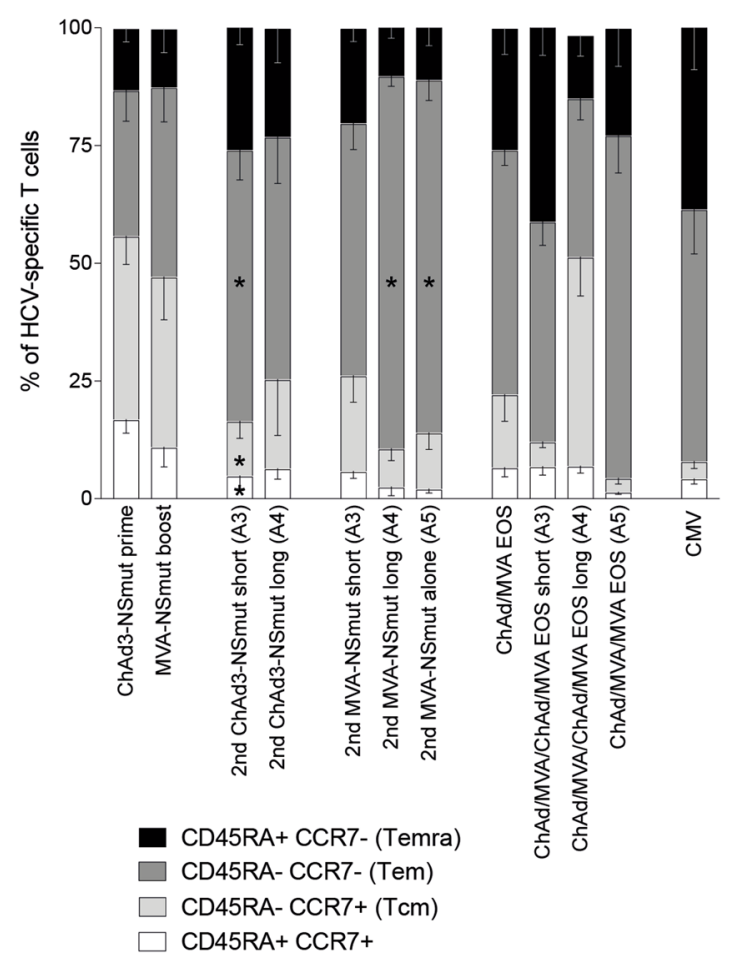

D

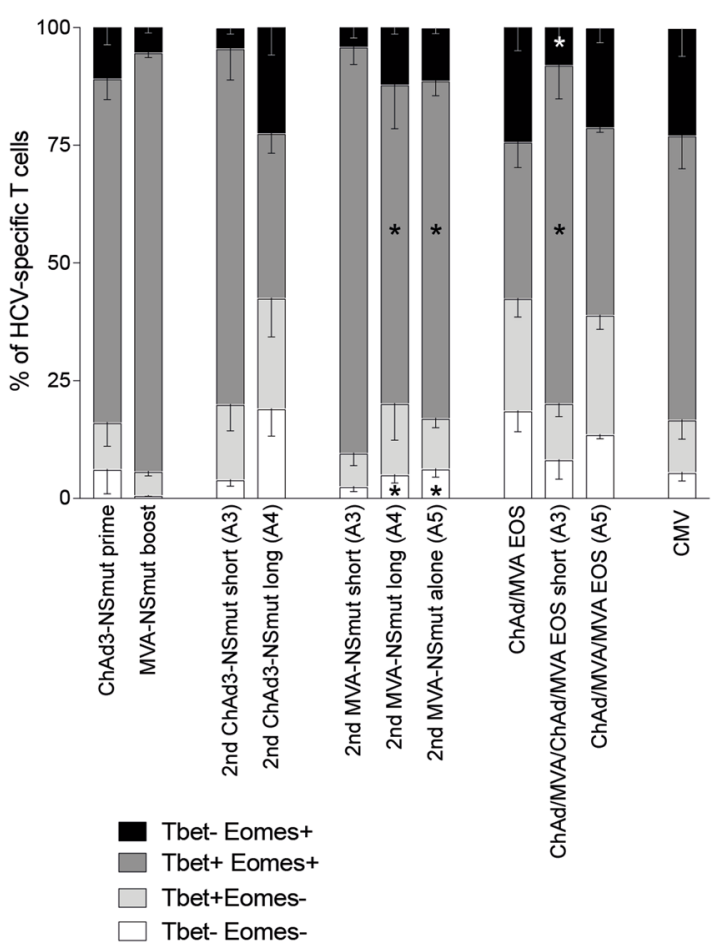

memory $\mathrm{T}$ cells elicited by different re-vaccination schedules. Although still the dominant population, after a second MVA vaccination fewer T cells were Tbet $^{+}$Eomes $^{+}$. It is important to note that even after reboosting $T$ cells were not driven to downregulate
Tbet whilst retaining Eomes, which is a TF profile indicative of T cell exhaustion by chronic viral infection (Fig. 6c, d) ${ }^{35}$.

Overall, ChAd-NSmut/MVA-NSmut induced T cells are predominantly effector memory similar to highly functional memory response to $\mathrm{CMV}$. The overall balance of $\mathrm{T}$ cell memory subsets is 
Fig. 6 Reboosting alters the proportions of $\mathbf{T}$ cell memory subsets. (a-d) PBMC from $\mathrm{HLA}-\mathrm{A}^{*} 01^{+}$or $\mathrm{A}^{*} 02^{+}$individuals were co-stained ex vivo with individual MHC class I pentamers and antibodies against surface markers CD45RA, CCR7 (a, b), or intranuclear transcription factors Tbet and Eomes (c, d). a Example FACS plots (pentamer gating and overlay of total CD8 ${ }^{+}$in grey and pentamer ${ }^{+}$in black; see

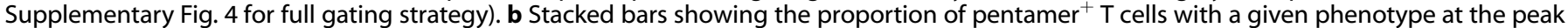
of the T cell response (1-4 weeks post vaccination, according to IFN $\gamma$ ELISpot) after each vaccination and at the end of the study (EOS; $n=$ 4-13 per pie). Where CMV responses were detected by IFN $\gamma$ ELISpot in HLA-A*02 ${ }^{+}$individuals an immunodominant CMV pentamer was used in parallel $(n=10)$. c Example FACS plots (overlay of total CD8 ${ }^{+}$in grey and pentamer ${ }^{+}$in black). d Stacked bars showing the proportion of pentamer $^{+}$T cells co-expressing Tbet and Eomes at the peak of the response (1-4 weeks post vaccination, according to IFN $\gamma$ ELISpot) after each vaccination and at the end of the study ( $n=4-13$ per pie). The proportion of CMV-specific T cells showing each subset phenotype is also shown $(n=6)$. b , d Mean, bars at standard error of mean. Kruskal-Wallis unpaired non-parametric Anova comparing ChAd3-NSmut prime vs. 2nd ChAd-NSmut short (A3) or long (A4), MVA-NSmut boost vs. 2nd MVA-NSmut short (A3) or long (A4) or alone (A5), ChAd3-NSmut/MVANSmut EOS vs. ChAd/MVA/CMVA EOS short (A3) or ChAd/MVA/MVA (A5). *P<0.05.

only subtly altered by repeated vaccination, and functionality and TF profile were not adversely affected by reboosting vaccination.

A ten-fold lower dose of MVA-NSmut can be used without impacting the magnitude, breadth, functionality, or phenotype of the vaccine-induced $T$ cell response

The possibility of inducing a comparable transgene-specific immune response whilst using a lower dose of MVA than is currently being used $\left(1-5 \times 10^{8} \mathrm{pfu}^{11,36-42}\right.$ would circumvent some of the difficulties and costs in manufacturing MVA and may reduce vaccine reactogenicity.

A dose de-escalation study was performed to assess the impact of lowering the MVA dose ten- or a hundred-fold on reactogenicity and immunogenicity. Healthy volunteers received a full priming dose of ChAd3-NSmut $\left(2.5 \times 10^{10} \mathrm{vp}\right)$ and then 8 weeks later volunteers received a medium dose of $2 \times 10^{7}$ pfu MVANSmut (arm A6) or a low dose of $2 \times 10^{6}$ pfu MVA-NSmut (arm A7). Following vaccination with a medium or low dose of MVANSmut very few AEs were observed (Supplementary Fig. 2). Only 11 and 7 AE's were experienced in the medium (all Grade 1) and low dose MVA arms respectively. A trend for a reduced number of AEs with lower doses of MVA-NSmut boosting vector was seen (Supplementary Table 4).

Importantly, no significant reduction in the HCV-specific T cell response to MVA-NSmut boost was observed at any time point during the trial when medium or low dose MVA-NSmut vaccinations are used (Fig. 7), although there is a trend for a lower peak and memory HCV-specific $T$ cell response with low dose MVA-NSmut (Fig. 7a, b). No reduction in the breadth of the vaccine-induced $T$ cell response (as determined by the number of positive pools in the IFNy ELISpot) at its peak or for the memory $T$ cell response was seen when lowering the dose of MVA-NSmut (Fig. 7c). The magnitude of the $T$ cell response to individual immunodominant HLA-A*01 and HLA-A*02 restricted epitopes was also comparable in volunteers who received a medium or low dose of MVA-NSmut, both at the peak of the response and at the end of the trial (Fig. 7d). T cells targeting these epitopes appeared to have a reduced expression of activation markers just after vaccination when given medium dose or low dose MVA-NSmut but showed comparable expression of cytolytic markers and PD-1 (Fig. 7e). HCV-specific T cells induced by lower doses of MVA also showed similar TF co-expression to high dose MVA vaccination (Fig. 7f). The cytokine production of $\mathrm{CD}^{+}$and $\mathrm{CD} 8^{+} \mathrm{HCV}$-specific $T$ cells on peptide stimulation at the peak of the $T$ cell response (TW9) was also comparable in those that received medium or low dose MVA-NSmut (Fig. 7g, h).

Finally, we assessed the ability of T cells induced by medium and low dose MVA vaccination to expand on further antigen encounter. $\mathrm{CD} 4^{+}$and $\mathrm{CD} 8^{+} \mathrm{T}$ cells induced by a medium dose of MVA had a robust in vitro proliferative response to HCV peptides that was comparable to T cells induced by high dose MVA (Fig. 8). The proliferative capacity of the T cells induced by all doses of MVA after stimulation with peptides covering the immunodominant pool (G, covering NS3 helicase) correlated well with the magnitude of the HCV-specific T cells response by ELISpot (Fig. 8); this suggests that despite the slightly lower magnitude of response (seen by ICS and ELISpot) there was no intrinsic proliferative defect in T cells induced by low dose MVA, as $\mathrm{T}$ cell proliferation was directly proportional to the magnitude of the response.

Overall, a hundred-fold reduction in MVA dose may impact the magnitude of the HCV-specific T cells response, however, a tenfold reduction in dose did not alter the magnitude, breadth, functionality, or phenotype of the vaccine-induced T cell response.

\section{DISCUSSION}

Viral vectored vaccines have become increasingly safe and efficacious as our understanding of the innate and adaptive immune responses to both the vector and the inserted transgene have developed, however, many questions remain as to how best to employ these potent $T$ cell inducers. To produce affordable, clinically practical, yet highly efficacious regimens optimisation of dose, timing, and vector combination is required.

Here we first investigated the possibility of enhancing the encoded transgene-specific memory $T$ cell response by reboosting volunteers that had previously received a prime-boost vaccination with ChAd3-NSmut/MVA-NSmut with further rounds of ChAd3NSmut and MVA-NSmut. As with single administrations, revaccination with ChAd3 or MVA was safe and well tolerated. The type, number, and severity of AEs were similar for first or second vaccinations with both ChAd3-NSmut or MVA-NSmut, and as with initial vaccination MVA was more reactogenic than ChAd3. Importantly, ChAd3 and MVA are no more reactogenic when readministered to the same healthy volunteers.

The activation status and the re-expansion of HCV-specific $\mathrm{T}$ cells was greatly limited when the second round of ChAd3NSmut/MVA-NSmut was given shortly after the initial prime boost (all vaccines 8 weeks apart). However, if reboosting is delayed strong recall responses are seen. When the second round of ChAdNSmut/MVA-NSmut was given a year after the initial prime-boost vaccinations the HCV-specific T cell response expanded to a similar magnitude as that seen at the peak after the initial vaccinations. We also showed that a simplified reboosting regimen employing a second MVA-NSmut alone appears to be as immunogenic as reboosting with ChAd3-NSmut/MVA-NSmut.

We assessed whether the re-expansion of $T$ cells targeting the HCV NS transgene, which was improved with a long interval between rounds of ChAd-NSmut, could be explained by the differential induction and maintenance of nAbs to $\mathrm{ChAd3}$, or through the HCV-specific T cell differentiation state at the time of administering the second ChAd3-NSmut vaccination. Anti-Ad vector $n A$ bs have been shown to limit transgene expression and $T$ cell induction after vaccination in mice and humans ${ }^{29,43-46}$ and extending the period between ChAd3-NSmut vaccinations may allow nAbs to wane and reduce anti-vector immunity. We found 
A

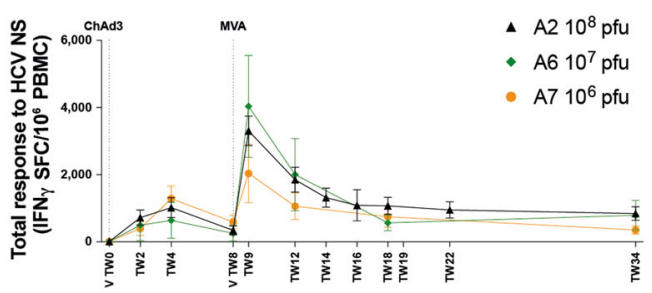

C

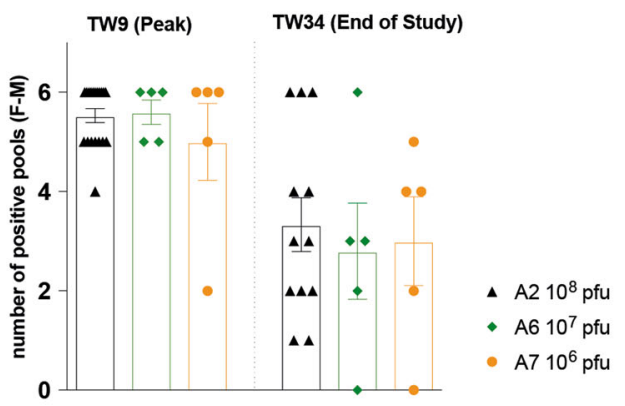

E

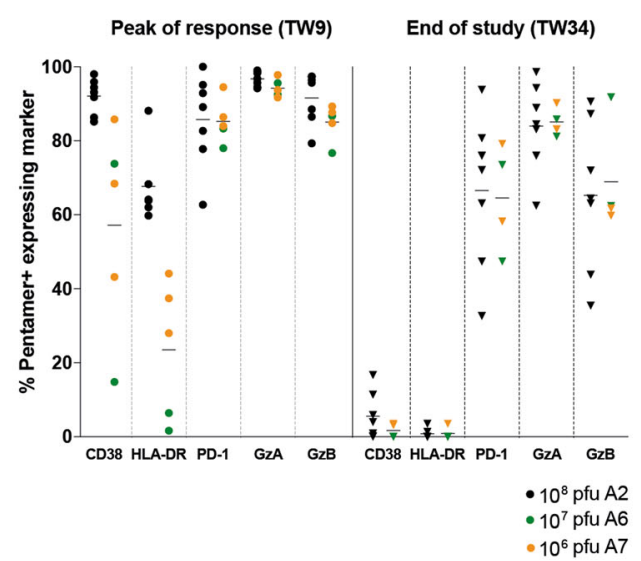

G

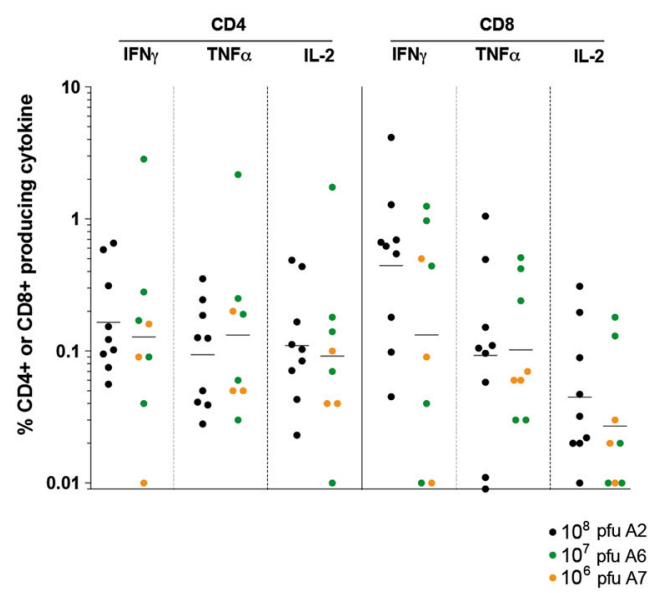

B

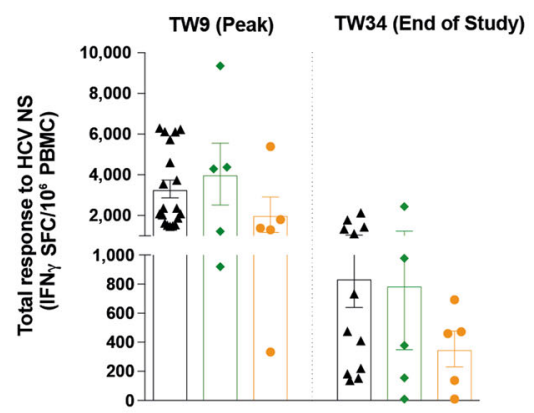

D

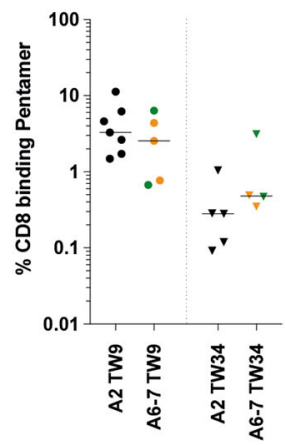

$\mathbf{F}$

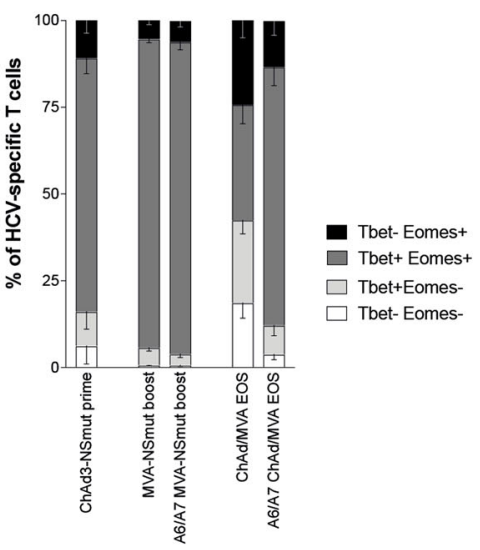

H

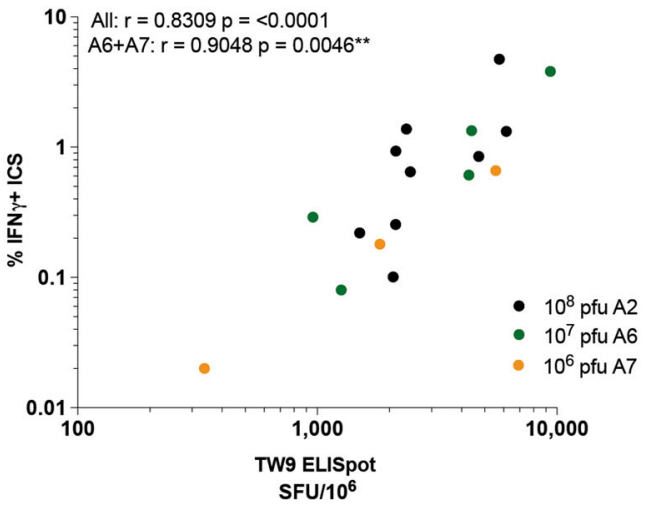

that pre-existing $\mathrm{nAb}$ titers to $\mathrm{ChAd} 3$ were low in all volunteers as expected, with no correlation between pre-existing nAb titers and vaccine immunogenicity. However, following the first ChAd vaccination, very high levels of $\mathrm{ChAd} 3 \mathrm{nAbs}$ were generated. Due to the small sample size, and the fact that by chance nAbs were higher at baseline in the short interval group (arm A3), it was not possible for us to directly conclude that a short interval between ChAd vaccinations directly resulted in higher nAbs at the time of the second ChAd vaccination. However, at these high levels of anti-Ad nAbs, there was a clear negative correlation 
Fig. 7 The magnitude, breadth, functionality, and phenotype of vaccine-induced HCV-specific T cells are unchanged when using a medium MVA-NSmut dose when compared to high dose. $\mathbf{a}-\mathbf{h}$ Healthy volunteers receiving ChAd3-NSmut prime vaccination were boosted with high ( $10^{8} \mathrm{pfu}$; black dots, arm A6), medium ( $10^{7} \mathrm{pfu}$; green dots, arm A7), or low dose (10 $0^{6}$ pfu; orange dots) MVA-NSmut vaccination 8 weeks later (Supplementary Table 1). a, b: the summed ex vivo IFN $\gamma$ ELISpot response to HCV NS encoded in the vaccine. a Kinetics of the HCV-specific T cell response across the vaccine trial (group mean). b-e Comparison of peak (1-week post-MVA-NSmut, TW9) and memory (end of study [EOS], TW34) (b) HCV-specific T cell response, (c) breadth of the HCV-specific T cell response (number of positive pools, see methods), (d) percentage of $\mathrm{CD}^{+} \mathrm{T}$ cells binding MHC class I pentamers ex vivo (NS3 ${ }_{1435-1443}, \mathrm{NS3}_{1406-1415}$ ), and (e) percentage of HCV-specific pentamer $^{+} \mathrm{T}$ cells expressing CD38, HLA-DR, PD-1, granzyme A (GzA) or granzyme B $(\mathrm{GzB})$. $f$ The percentage of pentamer ${ }^{+} \mathrm{T}$ cells coexpressing Tbet and Eomes at the peak of the T cell response after ChAd3-NSmut prime (TW2-4), after MVA-NSmut (TW9) and at EOS (arms A6 and A7 combined; TW34). g The percentage of CD4 ${ }^{+}$or CD8 ${ }^{+}$T cells producing IFN $\gamma$, TNF $\alpha$ or IL2 at the peak of the T cell response (TW9). h Correlation between the magnitude of HCV-specific T cell response induced by vaccination as measured by response to peptide pool $\mathrm{G}$ by ELISpot and percentage pentamer ${ }^{+}$(immunodominant epitope in pool G, HLA-A*02-restricted NS3 ${ }_{1406-1415}$ ). Spearman $r$ calculated for all data combined or for A6 and A7 data combined. a-c mean \pm standard error of mean. d, e, $\mathbf{g}$ Bars at median. b, c, e, $\mathbf{g}$ Kruskal-Wallis one-way Anova with Dunn's correction for multiple comparisons, all non-significant. d Mann-Whitney $t$ test non-significant.

A

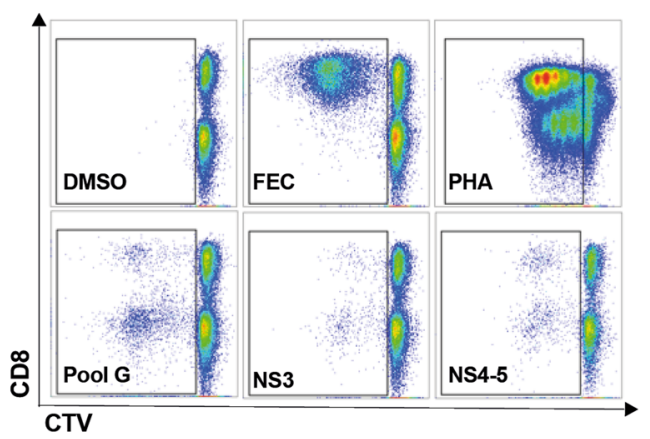

C

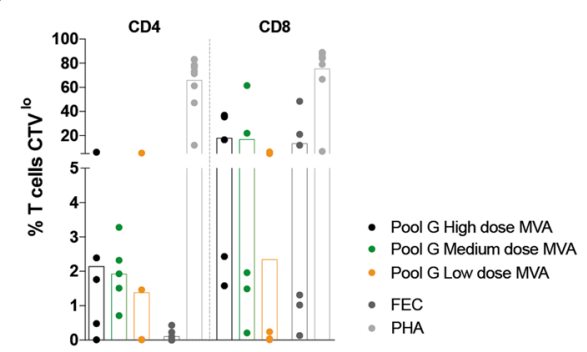

B

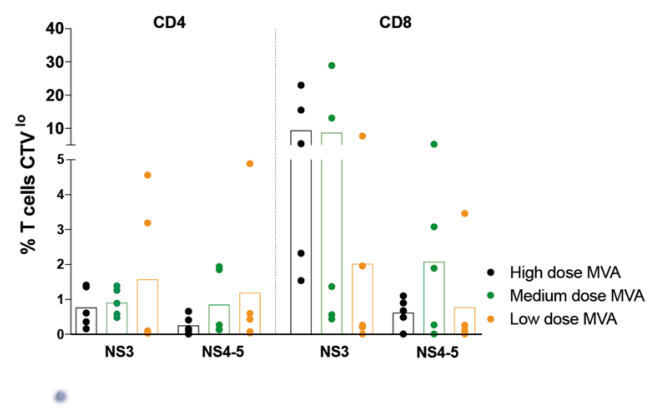

D

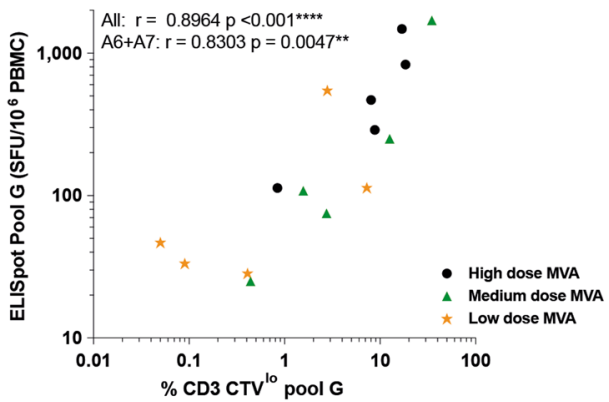

Fig. 8 Full and medium MVA-NSmut dose induced HCV-specific T cells with equivalent proliferative capacity. a Example plots of CTV dilution against $\mathrm{CD}^{+}$(gated on lymphocytes/singlets/live/CD3 ${ }^{+}$) for each stimulation (see Supplementary Fig. 4 for full gating strategy). b-d The percentage of $\mathrm{CD} 4^{+}$or $\mathrm{CD} 8^{+} \mathrm{T}$ cells that proliferated $\left(C T V^{\circ}\right)$ over 5 days in response to stimulation with (b) peptides covering the NS region of HCV (NS3 or NS4-5), (c) with a single immunodominant pool covering part of NS3h (pool G), with immunodominant MHC-class I restricted peptides from Flu, EBV, and CMV (FEC) or PHA (phytohemagglutinin). PBMC isolated at the end of the study were stimulated (11-32 weeks post-MVA-NSmut boost vaccination). d The percentage of $\mathrm{CD}^{+}{ }^{+}$T cells proliferating over 5 days in response to pool $\mathrm{G}$ was correlated with the T cell response to pool G by ELISpot at the same time point (end of study). b, c Kruskal-Wallis one-way Anova with Dunn's

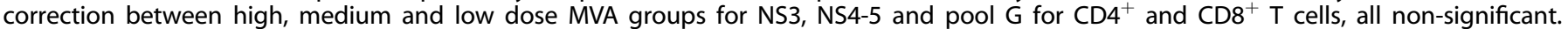
d Spearman $r$ calculated for all data combined or for A6 and A7 data combined.

between nAb Ad titer and the subsequent re-expansion of HCVspecific $T$ cells following the second ChAd3 vaccine. Therefore, future studies may evaluate $n A b s$ as a potential predictor of vaccine responsiveness in reboosting strategies. It is well recognized that $T$ cell response to adenovirus predominantly target the hexon protein and are cross-reactive amongst serotypes, as demonstrated by the vigorous expansion of antivector T cell responses upon vaccination detected by Ad5 Hexon stimulation here. However, there may be minor $\mathrm{T}$ cell responses to other viral proteins or ChAd3 hexon-specific $\mathrm{T}$ cell responses escaping our assessment. These limitations result in an imperfect ability to strictly determine the role and main players of anti- vector immunity in impacting the ability to respond to ChAd3 vector re-administration in our study.

The description of $\mathrm{T}$ cell memory subsets and the division of labor within a $\mathrm{T}$ cell response has led to the understanding that certain subsets are the main mediators of protection after vaccination and during the course of natural infection ${ }^{47-50}$. A longer interval between vaccinations may allow transgene-specific $T$ cells to fully differentiate into memory $T$ cells that can optimally respond to antigen re-exposure. We have previously observed that the HCV-specific T cells response contracts for at least 14 weeks after MVA-NSmut boost in most healthy volunteers and T cells continue to change phenotype and differentiate up to at least 26 weeks post-MVA-NSmut ${ }^{11}$. In volunteers who received ChAd3- 
NSmut/MVA-NSmut/ChAd3-NSmut/MVA-NSmut, all 8 weeks apart, $T$ cells assayed long term after the last vaccination showed a more terminally differentiated phenotype, whereas a larger population of $\mathrm{Tcm}$ persisted when reboosting was delayed. A large proportion of the HCV-specific T cells induced by all Ad and MVA containing regimens were Tem phenotype, a subset that is usually induced when $T$ cells see persistent low-level exposure to antigen ${ }^{31,51}$. However, CMV-derived vectors also induce predominantly Tem $\mathrm{CD}^{+} \mathrm{T}$ cells and promising efficacy studies in non-human primates of rhesus-derived CMV vectors encoding HIV- 1 antigens have prompted a paradigm shift to a preference for vaccine induction of circulating or tissue-resident memory $T$ cells with immediate effector function ${ }^{52-55}$. Reboosting after a long interval for $\mathrm{CD}^{+}$and $\mathrm{CD}^{+}{ }^{+} \mathrm{T}$ cells, and after a short interval for $\mathrm{CD} 8^{+}$ $T$ cells, also led to a larger proportion of polyfunctional transgenespecific $T$ cells than after the initial ChAd3-NSmut/MVA-NSmut prime/boost vaccinations. The $\mathrm{T}$ cell subset that will offer best protection against HCV infection is not yet known, however, this work highlights the exciting possibility of tailoring the timing and combinations of vectors within a regimen to elicit a specific mix of $T$ cell memory subsets.

We measured the expression of two transcription factors that coordinate many genes involved in differentiation and function of $\mathrm{CD}^{+} \mathrm{T}$ cells (Tbet and Eomes ${ }^{56-58}$ ). Tbet $^{+}$Eomes $^{-} \mathrm{CD}^{+}$ $T$ cells have been described as a progenitor population with an intrinsically low homeostatic turnover that give rise to Tbet $^{+}$Eomes $^{+}$cells when activated ${ }^{35}$. Repeated exposure to antigen above a certain threshold appears to strain the regenerative capacity of Tbet $^{+}$Eomes $^{-} \mathrm{T}$ cells, and drives the accumulation of exhausted Tbet $^{-}$Eomes $^{+}{ }^{+}$cells; this has been observed in the setting of chronic HBV, HCV, and HIV- $1^{35,59,60}$. It is important to note here that all vaccine regimens induced functional effector and memory $T$ cell responses, similar in phenotype to highly functional CMV-specific T cell memory ${ }^{31}$, which retained their Tbet expression, and there was no sign of exhaustion in functional assays or by TF expression.

Due to difficulties in manufacturing MVA vectors, a lower dose of MVA may be required for large-scale vaccine programs. A lower dose may also have the added benefit of reducing the reactogenicity of the vector and of lowering vaccine costs ${ }^{23}$. Here we perform the first in depth characterization of the transgenespecific $T$ cell response induced by a ten-fold $\left(10^{7} \mathrm{pfu}\right)$ and a $100-$ fold $\left(10^{6} \mathrm{pfu}\right)$ lower dose of MVA than that currently used in most phase I trials. We found that $10^{7}$ pfu MVA induces an equivalent T cell response to $10^{8}$ pfu MVA in terms of $T$ cell magnitude, breadth, kinetics, $\mathrm{T}$ cell phenotype (surface and transcription factor expression) and functionality (proliferative capacity, cytokine production, and cytolytic potential). Although not statistically significant, the magnitude of the HCV-specific T cell response may be reduced at its peak and at the end of the study when a 100fold lower dose $\left(10^{6} \mathrm{pfu}\right)$ is used. In addition, the number of AEs per vaccination was reduced for each severity, and percentage of individuals showing a given AE was lower for many AEs (e.g. moderate/severe pain, swelling, Erythema, Fever, Myalgia Fatigue etc.) when $10^{7}$ or $10^{6}$ pfu MVA was administered. This first-in-man dose titration of MVA heterologous boost, shows that a ten-fold reduction, compared to current standard dosing is equally immunogenic and better tolerated; a larger trial evaluating this dose of MVA is now required.

Several global health priorities (infectious agents and cancers) have shown an inherent ability to evade antibody vaccines and there is renewed optimism for the potential of $T$ cell-based vaccines ${ }^{55,61}$. Ad and MVA based vaccines are in development for most, if not all, of these health priorities and optimisation of T cell vaccine regimens is essential to aid in their development. In conclusion, we show that transgene-specific T cells responses can be boosted by further ChAd and MVA vaccinations and that the magnitude of the response is most effectively boosted when a long interval of at least 30 weeks is given between the initial vaccine round and reboosting. We also show that the phenotype of the vaccine-induced $T$ cells can be altered by repeat vaccinations. However, no signs of $T$ cell exhaustion are seen, even with the most intensive vaccine regimen. We also show importantly that a 10 -fold lower dose than is currently in testing for MVA vaccine vectors is as immunogenic and should be used in future trials.

\section{METHODS}

Ethics and regulatory approval

This clinical trial is registered in the ClinicalTrial.gov database (ID: NCT01296451, December 2010) and arms A1 and A2 of this clinical trial have been published previously in ${ }^{11}$. Approvals for the study were granted by the UK National Research Ethics Service, (NRES Committee South Central-Oxford REC A, REC reference number 10/H0604/45) and the UK Medicines and Healthcare Products Regulatory Agency (EudraCT no. 2009018260-10). GCP compliance was independently monitored by the University of Oxford Clinical Trials and Research Governance office. A multinational independent data safety monitoring committee (DSMC) provided safety oversight.

\section{Volunteer demographics and study arms}

Four volunteers in arm A2 were enrolled in to Arm A4 and received a second round of ChAd3-NSmut and MVA-NSmut vaccination 47-92 weeks after first receiving ChAd-NSmut prime vaccination (Fig. 1; Supplementary Table 1). The two primary endpoints of this study are vaccine safety and immunogenicity. All volunteers gave written informed consent before enrolment, and the studies were conducted according to the principles of the Declaration of Helsinki and in accordance with Good Clinical Practice. Volunteers were recruited at the CCVTM (Centre for Clinical Vaccinology and Tropical Medicine), Churchill Hospital, Oxford. Study arms and vaccination regimes are detailed (Supplementary Table 1). For this firstin-man Phase I study no randomisation or blinding of participants and data was used. A dose escalation for ChAd3-NSmut is described $\mathrm{in}^{62}$. The high MVA dose was selected on the basis of previous use of MVA vectors in human studies ${ }^{11,63,64}$. Vaccines were administered intramuscularly in the deltoid region of the arm.

Volunteers were observed for 1 to 3 hours after vaccination and then they systematically documented all symptoms and recorded a daily oral temperature. Solicited and un-solicited local and systemic AEs were collected in diary cards and recorded in case report forms. Both exposure to HCV (defined as HCV antibody and HCV RNA positivity) and recent intravenous drug use were study exclusion criteria. HCV antibodies, quantitative HCV RNA, and T cell response to HCV antigens were all undetectable at baseline. There were no signs or symptoms of viral hepatitis or an increase in liver enzymes during the study. One volunteer withdrew from follow up in arm A5 at trial week 14, prior to receiving reboosting vaccination with MVA-NSmut (volunteer 341). Safety data and immunogenicity data are included for this volunteer up to trial week 14.

\section{Vaccine constructs}

Non-replicative chimpanzee-derived adenoviral vaccine vectors (ChAd3) encoding the NS3-5B (NS) region of BK strain HCV (1985 amino-acids; accession number M58335; genotype $1 \mathrm{~b}$ ) has been described previously ${ }^{11,28,65}$. Generation of a MVA construct containing the same NS region transgene has been previously described ${ }^{11}$. The NS region contains a point mutation (GlyAspAsp to AlaAlaGly) at positions 1711 to 1713 in the catalytic site of the NS5b which inactivates this RNA polymerase without reducing the expression of HCV proteins or processing of the polypeptide by the NS3 encoded protease and is therefore referred to as NSmut ${ }^{66}$.

\section{Peptides and antigens}

494 peptides 15 amino-acids long overlapping by 11 amino-acids (BEI Resources) were divided into 6 pools (F-M) corresponding to NS3p, NS3h, NS4, NS5A, NS5B I, NS5B II (mean 82, range 73-112 peptides/pool) matching HCV genotype 1B strain BK. Peptides covering the complete sequence of the Hexon protein of Ad5 (15 amino-acids long overlapping by 11 amino-acids; UniProt ID: P04133; Miltenyi) were used to assess anti-vector immunity. Ad5 hexon and ChAd3 hexon sequence identity $87 \%$ and homology $91 \%$; $95 \%$ identity and $97 \%$ 
homology when excluding the hypervariable regions. Peptides were used at a final concentration of $3 \mu \mathrm{g} / \mathrm{ml}$ and $1 \mu \mathrm{g} / \mathrm{ml}$ per peptide for ELISpot and Intracellular cytokine staining, respectively.

\section{IFNY-ELISpot assays}

Unless otherwise stated culture medium for human T cells was R10: RPMI medium (GibcoBRL) supplemented with $10 \%$ by volume heat inactivated $\left(1 \mathrm{~h} 64^{\circ} \mathrm{C}\right)$ fetal calf serum (FCS; Hyclone, $1 \%$ by volume $200 \mathrm{mM} \mathrm{L}-$ glutamine (GibcoBRL) and $1 \%$ by volume $100 x$ penicillin and streptomycin solution (GibcoBRL).

IFNY-ELISpot assays were performed ex vivo in triplicate with $2 \times 10^{5}$ peripheral blood mononuclear cells (PBMC)/well in R10 as previously described ${ }^{62}$. PBMC were separated via density gradient (Lymphoprep) and counted using a Guava Personal Cell Analyser (Merck Millipore). Millipore AIP plates (MSIP54510) were coated at $4{ }^{\circ} \mathrm{C}$ with anti-human IFNy monoclonal antibody $(5 \mu \mathrm{g} / \mathrm{ml}$ in PBS; MAbTech clone 1-D1K) overnight. Plates were incubated for $3 \mathrm{~h}$ at room temperature with biotinylated mouse anti-human IFNy monoclonal antibody $(0.5 \mathrm{mg} / \mathrm{ml}$; MAbTech clone 7-B6-1) diluted in assay diluent (0.5\% BSA/PBS). The plate was then incubated with alkaline phosphatase-conjugated anti-biotin antibody diluted 1:750 in assay diluent (Vector Laboratories) for $2 \mathrm{~h}$ and finally incubated at room temperature with sterile filtered BCIP/NBT substrate solution (Pierce cat. 34042). Plates are air-dried overnight and read on an AID ELISpot plate reader. Internal controls were R10 alone (without cells) and DMSO (negative controls) and Concavalin A, FEC (HLA class I-restricted peptides from influenza, Epstein-Barr virus, and (MV), and CMV lysate (Virusys Corporation USA; positive controls).

A robust positive cut off for HCV-specific T cell responses in the IFNY-ELISpot assay was defined in ${ }^{62}$. For a positive response, the mean of antigen wells was determined (i) to be greater than $48 \mathrm{SFCs} / 10^{6}$ PBMCs and (ii) to exceed $3 \times$ background. Background wells (medium only, cells + DMSO) were typically zero to four spots. Total NS response was calculated by summing responses to all positive pools (NS3p-NS5B II) after correcting for background.

\section{Intracellular cytokine staining}

PBMC at $1 \times 10^{6}$ cells in $100 \mu \mathrm{l} \mathrm{R} 10$ were stimulated with peptide pools $(\mathrm{F}+\mathrm{G}+\mathrm{H}=\mathrm{NS} 3 / 4, \mathrm{I}+\mathrm{L}+\mathrm{M}=\mathrm{NS} 5 \mathrm{~A} / \mathrm{B}$ ) or PMA (phorbol 12-myristate 13acetate)/ionomycin (50 and $500 \mathrm{ng} / \mathrm{ml}$ respectively), or unstimulated (DMSO). Brefeldin-A was added $(10 \mu \mathrm{g} / \mathrm{ml}) 1 \mathrm{~h}$ later, cells were incubated overnight $\left(37^{\circ} \mathrm{C}\right)$, stained with fixable-NIR live/dead dye (Life Technologies), fixed (1\% paraformaldehyde) and permeabilised (Foxp3 Fix/Perm kit, $\mathrm{BD}$ Biosciences) then stained with the following antibodies at room temperature for 30 mins: CD3-PO (Pacific Orange; Invitrogen, UCHT1), CD4Qdot 605 (Invitrogen, S3.5) CD8-PB (Pacific Blue; BD biosciences, RPA-T8), IFNy-Alexa Fluor 700 (BD biosciences, XMG1.2), IL-2-APC (BD biosciences, 5344.111), and TNFa-PE-Cy7 (BD biosciences, MAb11). Flow cytometry was performed using a BD LSRII and analysis by FlowJo (Tree Star) version 10.4.1. All ICS data are corrected for background (cytokine production in paired DMSO wells).

Pestle version 1.8 and Spice version 6.0 were used for background subtraction, data formatting, and data visualization for polyfunctionality assessment of ICS data. Samples with a total T cell response to HCV NS of $<0.025 \%$ of $\mathrm{CD}^{+}$or $\mathrm{CD}^{+}{ }^{+}$T cells were excluded. Pie base, means.

FACS based proliferation assay-Cell Trace Violet dilution

To assess the proliferation of T cells after 5 -day stimulation with peptides or PHA, frozen PBMC were thawed and washed in PBS and 1-10 $\times 10^{6}$ PBMC were stained with $2.5 \mu \mathrm{M}$ Cell Trace Violet (ThermoFisher) for 10 mins in the dark at room temperature. 10x volume of ice cold $\mathrm{RH} 10$ (media containing 10\% human ab serum) was added and cells were kept on ice for 5 mins. PBMC were pelleted and resuspended in $10 \mathrm{mls}$ of $\mathrm{RH} 10$, transferred to a new tube and incubated at $37^{\circ} \mathrm{C}$ for 5 mins. PBMC were then washed again in PBS and plated $1 \times 10^{6}$ cells in $0.5 \mathrm{ml} \mathrm{RH} 10$ per well in a 48 well plate with the relevant stimulation; peptides covering HCV non-structural region were added at $1 \mu \mathrm{g} / \mathrm{ml}$ per peptide; immunodominant MHC-class I restricted peptides from Flu EBV and CMV (FEC) were added at $1 \mu \mathrm{g} / \mathrm{ml}$ per peptide; PHA (phytohemagglutinin) was added at $2 \mu \mathrm{g} / \mathrm{ml}$. After 5 days stimulation PBMC were harvested from the plate, washed in PBS, and were surface stained as above with fixable Live/dead and antibodies against CD3-FITC (Biolegend, UCHT1), CD4-Alexa fluor-700 (BD biosciences, RPA-T4) and CD8-PE-Cy7 (BD biosciences, RPA-T8) and were analyzed by flow cytometry as above.

\section{HLA Class I pentamer staining}

Frozen PBMC were thawed and stained ex vivo with $\mathrm{HLA} 0201$ or $H L A^{*} 0101$ PE-labelled pentamers (Proimmune Oxford; 20 min in PBS, RT), stained with LIVE/DEAD Fixable Near-IR Dead Cell Stain Kit (Thermo Fisher; 20 min RT), fixed (1\% formaldehyde in PBS, 20 mins RT) then stained with: CD3-PO (Pacific Orange; Invitrogen, UCHT1), CD8-PB (Pacific Blue; BD biosciences, RPA-T8), and either stained with CCR7-PeCy7 (CD197; BD biosciences, 3D12), CD45RAFITC (BD biosciences, HI100), CD38-PerCP-Cy5.5 (Biolegend, HIT2), HLA-DRAlexaFlour700 (BD biosciences, G46-6) for 30 min RT; or permeabilised (10x permeabilisation buffer; ebiosciences) and stained with PD1-PeCy7 (BD biosciences, EH12.1), Perforin-FITC (BE biosciences, dG9), Granzyme B- Alexa fluor700 (BD biosciences, GB11) and Granzyme A-PerCpCy5.5 (Biolegend, CB9) $30 \mathrm{~min}$ RT. For intranuclear staining, PBMC were stained as above for pentamers and Live/Dead dye, and then surface stained with: CD3-Pacificorange, CD8-PB (30 min RT). PBMC were then fixed (1 h RT) and permeabilised using the Foxp3/Transcription Factor Staining Buffer Set (ebiosciences; 45 min RT) and stained with eomes-eFluor660 (eBiosciences, WD1928) and TbetBV605 (Biolegend, 4B10).

\section{nAbs to ChAd3 vector}

An assay measuring the neutralising antibodies against $\mathrm{ChAd} 3$ vector was performed as previously described using recombinant ChAd3 expressing the reporter geneSEAP ${ }^{16,67}$.

\section{Statistical analysis}

Data were assumed to have a non-Gaussian distribution. Non-parametric tests were used throughout; paired tests within an individual (Wilcoxon matched pairs signed rank test) or unpaired tests between individuals (Mann-Whitney). For unpaired multiple comparisons Kruskal-Wallis oneway Anova with Dunn's correction was used. For correlations, Spearman's $r$ test was used. A $p$ value $<0.05$ was considered significant. Prism v. 7.0e (Graphpad) for Mac was used for analysis. ${ }^{*}=p \leq 0.05 ;{ }^{*}=p \leq 0.01$; ${ }^{* * *}=p \leq 0.001 ;{ }^{* * * *}=p<0.0001$.

\section{Reporting summary}

Further information on research design is available in the Nature Research Reporting Summary linked to this article.

\section{DATA AVAILABILITY}

The data that support the findings of this study are available from the corresponding author L.S. upon reasonable request. This study did not generate any unique code or datasets other than those included in this published article (and its Supplementary Information files).

Received: 26 March 2020; Accepted: 20 August 2020; Published online: 12 October 2020

\section{REFERENCES}

1. Fougeroux, C. \& Holst, P. J. Future prospects for the development of cost-effective adenovirus vaccines. Int. J. Mol. Sci. 18, 686 (2017).

2. Vitelli, A. et al. Chimpanzee adenoviral vectors as vaccines-challenges to move the technology into the fast lane. Expert Rev. Vaccines 16, 1241-1252 (2017).

3. Meyer, H., Sutter, G. \& Mayr, A. Mapping of deletions in the genome of the highly attenuated vaccinia virus MVA and their influence on virulence. J. Gen. Virol. 72, 1031-1038 (1991)

4. López-Camacho, C. et al. Rational Zika vaccine design via the modulation of antigen membrane anchors in chimpanzee adenoviral vectors. Nat. Commun. 9, 2441 (2018).

5. Ragonnaud, E. et al. Replication deficient human adenovirus vector serotype 19a/ 64: Immunogenicity in mice and female cynomolgus macaques. Vaccine 36, 6212-6222 (2018)

6. Bliss, C. M. et al. Assessment of novel vaccination regimens using viral vectored liver stage malaria vaccines encoding ME-TRAP. Sci. Rep. 8, 3390 (2018).

7. Coughlan, L. et al. Heterologous two-dose vaccination with simian adenovirus and poxvirus vectors elicits long-lasting cellular immunity to influenza virus $A$ in healthy adults. EBioMedicine 29, 146-154 (2018).

8. Rampling, T. et al. Safety and efficacy of novel malaria vaccine regimens of RTS,S/ AS01B alone, or with concomitant ChAd63-MVA-vectored vaccines expressing ME-TRAP. npj Vaccines 3, 49 (2018). 
9. Folegatti, P. M. et al. Safety and immunogenicity of a candidate Middle East respiratory syndrome coronavirus viral-vectored vaccine: a dose-esca lation, open-label, non-randomised, uncontrolled, phase 1 trial. Lancet Infect. Dis. https:// doi.org/10.1016/S1473-3099(20)30160-2 (2020).

10. van Doremalen, $\mathrm{N}$. et al. ChAdOx1 nCoV-19 vaccination prevents SARS-CoV-2 pneumonia in rhesus macaques. bioRxiv https://doi.org/10.1101/2020.05.13.093195 (2020).

11. Swadling, L. et al. A human vaccine strategy based on chimpanzee adenoviral and MVA vectors that primes, boosts, and sustains functional HCV-specific T cell memory. Sci. Transl. Med. 6, 1-8 (2014).

12. Antrobus, R. D. et al. Coadministration of seasonal influenza vaccine and MVA-NP $+\mathrm{M} 1$ simultaneously achieves potent humoral and cell-mediated responses. Mol. Ther. 22, 233-238 (2014).

13. Ewer, K. et al. A monovalent chimpanzee adenovirus ebola vaccine boosted with MVA. N. Engl. J. Med. 374, 1635-1646 (2016).

14. Green, C. A. et al. Chimpanzee adenovirus- and MVA-vectored respiratory syncytial virus vaccine is safe and immunogenic in adults. Sci. Transl. Med. 7, 300ra126-300ra126 (2015).

15. Gómez, C. E. et al. A phase i randomized therapeutic MVA-B vaccination improves the magnitude and quality of the T cell immune responses in HIV-1-infected subjects on HAART. PLOS ONE 10, e0141456 (2015)

16. Hartnell, F. et al. A novel vaccine strategy employing serologically different chimpanzee adenoviral vectors for the prevention of HIV-1 and HCV coinfection. Front. Immunol. 10, 3175 (2019).

17. Stanley, D. A. et al. Chimpanzee adenovirus vaccine generates acute and durable protective immunity against ebolavirus challenge. Nat. Med. 20, 1126-1129 (2014).

18. Royle, J. \& Buttery, J. Immunisation. Paediatr. Handb. Eighth Ed. 4, 110-124 (2009).

19. Borducchi, E. N. et al. Ad26/MVA therapeutic vaccination with TLR7 stimulation in SIV-infected rhesus monkeys. Nature 540, 284-287 (2016).

20. Taylor, G. et al. Efficacy of a virus-vectored vaccine against human and bovine respiratory syncytial virus infections. Sci. Transl. Med. 7, 300ra127-300ra127 (2015).

21. O'Hara, G. A. et al. Clinical assessment of a recombinant simian adenovirus ChAd63: A potent new vaccine vector. J. Infect. Dis. 205, 772-781 (2012).

22. Moorthy, V. S. et al. Phase 1 evaluation of 3 highly immunogenic prime-boost regimens, including a 12-month reboosting vaccination, for malaria vaccination in Gambian men. J. Infect. Dis. 189, 2213-2219 (2004).

23. Cottingham, M. G. \& Carroll, M. W. Recombinant MVA vaccines: dispelling the myths. Vaccine 31, 4247-4251 (2013).

24. Leen, A. M. et al. Identification of hexon-specific CD4 and CD8 T-cell epitopes for vaccine and immunotherapy. J. Virol. 82, 546-554 (2008).

25. Leen, A. M. et al. Conserved CTL epitopes on the adenovirus hexon protein expand subgroup cross-reactive and subgroup-specific $\mathrm{CD}^{+} \mathrm{T}$ cells. Blood 104, 2432-2440 (2004).

26. Onion, D. et al. The CD4 ${ }^{+} \mathrm{T}$-cell response to adenovirus is focused against conserved residues within the hexon protein. J. Gen. Virol. 88, 2417-2425 (2007).

27. Hutnick, N. A. et al. Adenovirus-specific human T cells are pervasive, polyfunctional, and cross-reactive. Vaccine 28, 1932-1941 (2010).

28. Colloca, S. et al. Vaccine vectors derived from a large collection of simian adenoviruses induce potent cellular immunity across multiple species. Sci. Transl. Med. 4, 115ra2-115ra2 (2012).

29. Zak, D. E. et al. Merck Ad5/HIV induces broad innate immune activation that predicts $\mathrm{CD}^{+}{ }^{+}$-cell responses but is attenuated by preexisting Ad5 immunity. Proc. Natl Acad. Sci. U. S. A. 109, E3503-E3512 (2012).

30. Sallusto, F., Geginat, J. \& Lanzavecchia, A. Central memory and effector memory t cell subsets: function, generation, and maintenance. Annu. Rev. Immunol. 22, 745-763 (2004)

31. Bolinger, B. et al. Adenoviral vector vaccination induces a conserved program of $\mathrm{CD}^{+} \mathrm{T}$ cell memory differentiation in mouse and man. Cell Rep. 13, 1578-1588 (2015).

32. Cruz-Guilloty, F. et al. Runx3 and T-box proteins cooperate to establish the transcriptional program of effector CTLs. J. Exp. Med. 206, 51-59 (2009)

33. Takemoto, N., Intlekofer, A. M., Northrup, J. T., Wherry, E. J. \& Reiner, S. L. Cutting edge: IL-12 inversely regulates T-bet and eomesodermin expression during pathogen-induced $\mathrm{CD}^{+} \mathrm{T}$ cell differentiation. J. Immunol. 177, 7515-7519 (2006)

34. Intlekofer, A. M. et al. Effector and memory $\mathrm{CD}^{+} \mathrm{T}$ cell fate coupled by T-bet and eomesodermin. Nat. Immunol. 6, 1236-1244 (2005).

35. Paley, M. A. et al. Progenitor and terminal subsets of $\mathrm{CD}^{+} \mathrm{T}$ cells cooperate to contain chronic viral infection. Science 338, 1220-1225 (2012).

36. Zeng, L. S. et al. Emplacement depth of the Shidao granitic complex and the rapid exhumation of the Sulu ultrahigh pressure rocks: New constraints on the mechanisms for rapid exhumation. Acta Petrol. Sin. 23, 3171-3179 (2007).
37. Ndiaye, B. P. et al. Safety, immunogenicity, and efficacy of the candidate tuberculosis vaccine MVA85A in healthy adults infected with HIV-1: a randomised, placebo-controlled, phase 2 trial. Lancet Respir. Med. 3, 190-200 (2015).

38. Todryk, S. M. et al. The relationship between human effector and memory $\mathrm{T}$ cells measured by ex vivo and cultured ELISPOT following recent and distal priming. Immunology 128, 83-91 (2009).

39. Ogwang, C. et al. Safety and immunogenicity of heterologous prime-boost immunisation with plasmodium falciparum malaria candidate vaccines, ChAd63 ME-TRAP and MVA ME-TRAP, in healthy Gambian and Kenyan adults. PLOS ONE 8 , e57726 (2013).

40. Sheehy, S. H. et al. Phase la clinical evaluation of the plasmodium falciparum blood-stage antigen MSP1 in ChAd63 and MVA vaccine vectors. Mol. Ther. 19, 2269-2276 (2011).

41. Overton, E. T. et al. Safety and immunogenicity of modified vaccinia AnkaraBavarian Nordic smallpox vaccine in vaccinia-naive and experienced human immunodeficiency virus-infected individuals: An open-label, controlled clinical phase II trial. Open Forum Infect. Dis. 2, ofv040 (2015).

42. Kreijtz, J. H. C. M. et al. Safety and immunogenicity of a modified-vaccinia-virusAnkara-based influenza A H5N1 vaccine: a randomised, double-blind phase 1/2a clinical trial. Lancet Infect. Dis. 14, 1196-1207 (2014).

43. Steffensen, M. A. et al. Pre-existing vector immunity does not prevent replication deficient adenovirus from inducing efficient cd8 t-cell memory and recall responses. PLoS ONE 7, e34884 (2012).

44. Sumida, S. M. et al. Neutralizing antibodies and $\mathrm{CD}^{+} \mathrm{T}$ lymphocytes both contribute to immunity to adenovirus serotype 5 vaccine vectors. J. Virol. 78 2666-2673 (2004).

45. Fitzgerald, J. C. et al. A simian replication-defective adenoviral recombinant vaccine to HIV-1 Gag. J. Immunol. 170, 1416-1422 (2003).

46. Youil, R. et al. Hexon gene switch strategy for the generation of chimeric recombinant adenovirus. Hum. Gene Ther. 13, 311-320 (2002).

47. Hansen, S. G. et al. Erratum: effector memory T cell responses are associated with protection of rhesus monkeys from mucosal simian immunodeficiency virus challenge (Nature Medicine (2009) 15 (293-299)). Nat. Med. 15, 462 (2009).

48. Barouch, D. H. et al. Vaccine protection against acquisition of neutralizationresistant SIV challenges in rhesus monkeys. Nature 482, 89-93 (2012).

49. Northfield, J. W. et al. Human immunodeficiency virus type 1 (HIV-1)-specific CD8 ${ }^{+}$TEMRA cells in early infection are linked to control of HIV-1 viremia and predict the subsequent viral load set point. J. Virol. 81, 5759-5765 (2007).

50. Sridhar, S. et al. Cellular immune correlates of protection against symptomatic pandemic influenza. Nat. Med. 19, 1305-1312 (2013).

51. Gordon, C. L. et al. Induction and maintenance of CX3CR1-intermediate peripheral memory CD8+ T cells by persistent viruses and vaccines. Cell Rep. 23 768-782 (2018).

52. Picker, L. J. Are effector memory T cells the key to an effective HIV/AIDS vaccine? EMBO Rep. 15, 820-821 (2014).

53. Makedonas, G. \& Betts, M. R. Living in a house of cards: Re-evaluating CD8 ${ }^{+}$T-cell immune correlates against HIV. Immunol. Rev. 239, 109-124 (2011).

54. Hansen, S. G. et al. Profound early control of highly pathogenic SIV by an effector memory T-cell vaccine. Nature 473, 523-527 (2011).

55. McMichael, A. J. Is a human CD8 T-cell vaccine possible, and if so, what would it take?: Could a CD8 ${ }^{+}$T-cell vaccine prevent persistent HIV infection? Cold Spring Harb. Perspect. Biol. 10, a029124 (2018).

56. Doering, T. A. et al. Network analysis reveals centrally connected genes and pathways involved in $\mathrm{CD}^{+} \mathrm{T}$ cell exhaustion versus memory. Immunity $\mathbf{3 7}$ 1130-1144 (2012).

57. Smith, C. et al. Endogenous antigen presentation impacts on T-box transcription factor expression and functional maturation of $\mathrm{CD}^{+} \mathrm{T}$ cells. Blood 120 3237-3245 (2012).

58. Rao, R. R., Li, Q., Odunsi, K. \& Shrikant, P. A. The mTOR KINASE DETERMINES EFFECTOR VERSUS MEmory $\mathrm{CD}^{+} \mathrm{t}$ cell fate by regulating the expression of transcription factors T-bet and eomesodermin. Immunity 32, 67-78 (2010).

59. Ribeiro-dos-Santos, P. et al. Chronic HIV infection affects the expression of the 2 transcription factors required for CD8 T-cell differentiation into cytolytic effectors. Blood 119, 4928-4938 (2012).

60. Kao, C. et al. Transcription factor T-bet represses expression of the inhibitory receptor PD-1 and sustains virus-specific $\mathrm{CD}^{+}{ }^{+} \mathrm{T}$ cell responses during chronic infection. Nat. Immunol. 12, 663-671 (2011).

61. Beura, L. K., Jameson, S. C. \& Masopust, D. Is a human CD8 T-cell vaccine possible, and if so, what would it take?: CD8 T-cell vaccines: To B or not to B? Cold Spring Harb. Perspect. Biol. 10, a028910 (2018).

62. Barnes, E. et al. Novel adenovirus-based vaccines induce broad and sustained $T$ cell responses to HCV in man. Sci. Transl. Med. 4, 115ra1-115ra1 (2012).

63. Sheehy, S. H. et al. ChAd63-MVA-vectored blood-stage malaria vaccines targeting MSP1 and AMA1: Assessment of efficacy against mosquito bite challenge in humans. Mol. Ther. 20, 2355-2368 (2012). 
64. Porter, D. W. et al. A human phase I/lla malaria challenge trial of a polyprotein malaria vaccine. Vaccine 29, 7514-7522 (2011).

65. Folgori, A. et al. A T-cell HCV vaccine eliciting effective immunity against heterologous virus challenge in chimpanzees. Nat. Med. 12, 190-197 (2006).

66. Capone, S. et al. A novel adenovirus type 6 (Ad6)-based hepatitis $C$ virus vector that overcomes preexisting Anti-Ad5 immunity and induces potent and broad cellular immune responses in Rhesus Macaques. J. Virol. 80, 1688-1699 (2006).

67. Aste-Amézaga, M. et al. Quantitative adenovirus neutralization assays based on the secreted alkaline phosphatase reporter gene: application in epidemiologic studies and in the design of adenovector vaccines. Hum. Gene Ther. 15, 293-304 (2004).

\section{ACKNOWLEDGEMENTS}

Supported by the Medical Research Council (MRC) UK and the European Union (Framework Vl; HEPACIVAC) by funding the study and the manufacture of MVANSmut through an MRC UK DCS (Developmental Clinical Studies) award (G0701694). Financial support also supplied by GSK. P.K. is supported by the Wellcome Trust, the Oxford NHIR BRC and the U19 grant (2U19AI082630-06). L.S. was supported by an MRC CASE studentship and Medical Research Foundation research grant (MRF-0440001-RG-SWADL). E.B. was funded as an MRC Senior Clinical Scientist, and is supported by the Oxford NHIR BRC, and the Jenner Institute. The authors would like to acknowledge $\mathrm{BEI}$ resources for providing peptides.

\section{AUTHOR CONTRIBUTIONS}

E.B., S.Ca, A.F., A.N., R.C., S.Co., C.T., V.V., designed the study/protocols and contributed to scientific discussion. L.S., F.H, A.B., S.Ca., M.D.S., A.F., E.B., and P.K. performed the research and analysis. L.S., E.B., A.F., S.Ca., and P.K. wrote the manuscript. E.B. was the principal investigator.

\section{COMPETING INTERESTS}

S.Co., A.F., R.C., and A.N. are named inventors on patent applications covering HCVvectored vaccines and chimpanzee adenovirus vectors [WO 2006133911 (A3) hepatitis C virus nucleic acid vaccine, WO 2005071093 (A3) chimpanzee adenovirus vaccine carriers, WO 03031588 (A2) hepatitis C virus vaccine]. V.V. is an employee of GSK group of companies. The rest of the authors declare that there are no competing interests.

\section{ADDITIONAL INFORMATION}

Supplementary information is available for this paper at https://doi.org/10.1038/ s41541-020-00240-0.

Correspondence and requests for materials should be addressed to L.S.

Reprints and permission information is available at http://www.nature.com/ reprints

Publisher's note Springer Nature remains neutral with regard to jurisdictional claims in published maps and institutional affiliations.

(i) Open Access This article is licensed under a Creative Commons Attribution 4.0 International License, which permits use, sharing, adaptation, distribution and reproduction in any medium or format, as long as you give appropriate credit to the original author(s) and the source, provide a link to the Creative Commons license, and indicate if changes were made. The images or other third party material in this article are included in the article's Creative Commons license, unless indicated otherwise in a credit line to the material. If material is not included in the article's Creative Commons license and your intended use is not permitted by statutory regulation or exceeds the permitted use, you will need to obtain permission directly from the copyright holder. To view a copy of this license, visit http://creativecommons. org/licenses/by/4.0/.

(c) The Author(s) 2020 